\title{
Improvement of Optical Tracking-Based Orientation Estimation by Fusing Gyroscope Information
}

\author{
Zhicheng Yang ${ }^{\circledR}$, Shenggang Yan ${ }^{\circledR}$, Bert-Jan F. van Beijnum ${ }^{\circledR}$, Member, IEEE, Bin Li ${ }^{\circledR}$, \\ and Peter H. Veltink $\left.{ }^{(}\right)$, Senior Member, IEEE
}

\begin{abstract}
Optical tracking systems (OTS) can provide high position accuracy over a large workspace. However, the orientation from the OTS is related to the distance between markers, which contains large errors when the distance is small or line-ofsight problems occur. The orientation estimation with a gyroscope is complementary to the OTS-based orientation. In this article, an error-state Kalman filter (ESKF) is proposed to fuse them. Two experiments were performed to verify the performance: first, a unit including markers and a gyroscope was placed statically and rotated dynamically in 3-D space. Secondly, two units were used to estimate the relative orientation between the hand and fingers. The static and dynamic orientation errors reduced from $0.39^{\circ} \pm 0.16^{\circ}$ and $2.75^{\circ} \pm 1.56^{\circ}$ to $0.23^{\circ} \pm 0.02^{\circ}$ and $1.50^{\circ} \pm 0.62^{\circ}$, respectively, when the distance between markers was $13 \mathrm{~mm}$. The second experimental results show that the fused method improved the OTS performance by smoothing the estimate, filling the relative orientation during the line of sight period, and correcting the estimation when there were identification problems of markers.
\end{abstract}

Index Terms-Data fusion, gyroscope, optical tracking system (OTS), orientation.

\section{INTRODUCTION}

$\mathbf{O}$ PTICAL tracking systems (OTS) have become increasingly powerful tools in many biomedical areas, such as biomechanics, gait analysis, and sports performance evaluation [1]-[3]. An OTS is composed of a set of high-speed cameras and reflective markers or infrared emitting diode markers. Compared with alternative motion tracking systems, such as electromagnetic systems and systems based on inertial measurement units (IMU) or inertial and magnetic measurement units (IMMU), the biggest advantage of OTS is high position estimation accuracy. Therefore, OTS is often used as "gold standard" for position estimation [4]-[6]. However, OTS requires a cluster of at least three markers on a body segment for 3-D orientation estimation. The cluster determines

Manuscript received September 29, 2020; revised March 8, 2021; accepted April 4, 2021. Date of publication April 14, 2021; date of current version May 5, 2021. The work of Z. Yang was supported by the China Scholarship Council. The Associate Editor coordinating the review process was Yuya Koyama. (Corresponding author: Zhicheng Yang.)

Zhicheng Yang is with the School of Marine Science and Technology, Northwestern Polytechnical University, Xi'an 710072, China, and also with the Department of Biomedical Signals and Systems, University of Twente, 7522 NB Enschede, The Netherlands.

Shenggang Yan and Bin Li are with the School of Marine Science and Technology, Northwestern Polytechnical University, Xi'an 710072, China.

Bert-Jan F. van Beijnum and Peter H. Veltink are with the Department of Biomedical Signals and Systems, University of Twente, 7522 NB Enschede, The Netherlands.

Digital Object Identifier 10.1109/TIM.2021.3073293 a frame of relative marker positions in 3-D space and thus a 3-D orientation. The accuracy of such OTS-based orientation estimation is limited by two factors, being the relative position accuracy and line-of-sight occlusion. The relative marker position accuracy depends on the accuracy of the marker position estimates and the distances between the markers. The orientation estimate for a body segment that is large and rigid can be adequately accurate since the marker cluster can be spatially extended. However, OTS-based orientation estimation may be inaccurate if body segments are nonrigid or small, as is the case for hand and fingers. The second factor which may impede OTS-based 3-D orientation estimation is "line of sight" problems due to occlusion.

An alternative popular option for orientation estimation is an IMMU-based system [7]-[9]. Rate gyroscope, contained in an IMMU, measure angular velocity directly, from which change of orientation over time can be determined by integration over time. Such estimation can only be trustful over a short period of time because of integration drift. Full 3-D orientation can be estimated and integration drift canceled if orientation information from angular velocity sensors is combined with complementary information provided by accelerometers and magnetometers, which are also contained in IMMU systems. In this manner, a rather accurate orientation estimate without drift can be obtained [10], [11]. Heading of IMMU-based 3-D orientation estimation can, however, easily be disturbed by magnetic disturbances that may especially occur in indoor environments [12], [13]. In addition, inclination estimates may be disturbed if nongravitational acceleration is not negligible compared with gravitational acceleration. Finally, unlike OTS, estimates of change of 3-D position using IMMU systems show large drift over time due to the integration operations involved in strapdown navigation, while position estimation is often important in addition to orientation estimation [14]-[16].

Fusion of the OTS and IMMU-based movement analysis may improve 3-D orientation estimation. It may also potentially help in filling occlusion gaps in the OTS-based orientation estimation. Arash et al. [17] alternatively proposed an orientation-based fusion of inertial and Microsoft Kinect sensors for this purpose. Microsoft Kinect sensors exploit a depth camera rather than high-speed cameras and optical markers. The depth information can be used to reconstruct the shape of the object, which can help to estimate the orientation [18]-[20]. The advantages of using Kinect sensors are low cost and no marker attachment. However, its accuracy is still not 


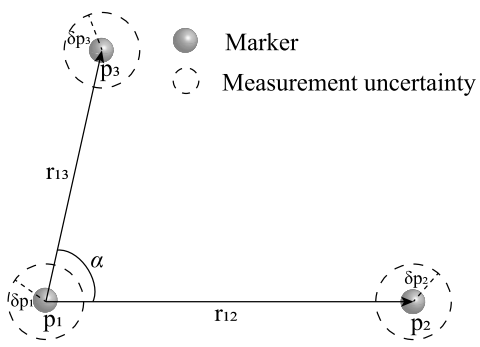

Fig. 1. Illustration of a marker cluster used for orientation estimation with measurement errors. The positions of markers are $\boldsymbol{p}_{i}(i=1,2,3)$ with standard deviation $\sigma_{m}$, which will cause error for relative position $\boldsymbol{r}_{12}$ and $\boldsymbol{r}_{13}$, and subsequently cause errors for the orientation estimation.

comparable with marker-based OTS. He et al. [21] proposed a 6 DOF motion tracking method based on IMMU and OTS with an augmented reality head-mounted display (ARHMD). They used the position from OTS and orientation estimate from an IMMU to update the estimated position of each OTS marker and, subsequently improve the orientation estimation. The accuracy, in this case, is closely related to the orientation estimation errors caused by the nongravitational acceleration and the magnetic disturbance.

In this article, we concentrate on improving the accuracy of the OTS-based orientation estimation and filling the orientation data during occlusions. This is of primary interest in many applications, such as hand movement tracking for rehabilitation [22], [23] and navigated lateral skull base surgeries [24]. We propose to estimate 3-D orientation by fusing the information from an OTS and a rate gyroscope, without including the information from the accelerometer and the magnetometer in order to avoid the errors they may induce: First, the error caused by the integration drift of linear accelerations extracted from the accelerometer in case change of occluded marker positions would be estimated is eliminated, since only orientation estimates from OTS and gyroscope are fused. Secondly, the orientation errors induced by the nongravitational acceleration and the magnetic disturbance are eliminated. We demonstrated our proposed method through experimental evaluation.

\section{Methods}

In this section, we first describe the orientation error induced from an OTS, and subsequently a gyroscope sensor model and orientation estimation using the gyroscope. Finally, we propose an error-state Kalman filter (ESKF) and smoothing techniques to fuse the data from the OTS and the gyroscope sensor. For the description below, three different coordinate frames are used.

Sensor frame, $\mathrm{s}$ - determined by the gyroscope sensor;

Marker frame, $\mathrm{m}$-determined by the optical markers;

Global frame, g-determined by the calibration of the optical system prior to the experiment. Positions of the markers are measured in this frame and also the quaternion-based orientation is determined relative to this frame.

\section{A. Error Analysis of Orientation Estimation Using an OTS}

Orientation estimation using OTS is commonly based on position measurements of a cluster of markers. As shown in Fig. 1, positions of three markers are measured as

$$
\left\{\begin{array}{l}
\boldsymbol{y}_{m i}^{g}=\boldsymbol{p}_{i}^{g}+\delta \boldsymbol{p}_{i}^{g} \quad(i=1,2,3) \\
\delta \boldsymbol{p}_{i}^{g} \sim\left(0, \sigma_{m}\right)
\end{array}\right.
$$

where $\boldsymbol{p}_{i}^{g}$ is the true position of the marker and $\delta \boldsymbol{p}_{i}^{g}$ is the measurement error, which is assumed to have a normal distribution with zero mean and standard deviation $\sigma_{m} \cdot \boldsymbol{y}_{m i}^{g}$ is the marker position measured by the OTS. All parameters are in the global frame. The relative position between markers is calculated as

$$
\hat{\boldsymbol{r}}_{i j}^{g}=\boldsymbol{y}_{m j}^{g}-\boldsymbol{y}_{m i}^{g}=\boldsymbol{r}_{i j}^{g}+\delta \boldsymbol{p}_{i j}^{g} \quad(j=2,3 ; i=1)
$$

where $\hat{\boldsymbol{r}}_{i j}^{g}$ is the relative position obtained from OTS measurements, $\boldsymbol{r}_{i j}^{g}$ is the true relative position, $\delta \boldsymbol{p}_{i j}^{g}$ is the measurement error

$$
\left\{\begin{array}{l}
\boldsymbol{r}_{i j}^{g}=\boldsymbol{p}_{j}^{g}-\boldsymbol{p}_{i}^{g} \\
\delta \boldsymbol{p}_{i j}^{g}=\delta \boldsymbol{p}_{j}^{g}-\delta \boldsymbol{p}_{i}^{g}, \quad \delta \boldsymbol{p}_{i j}^{g} \sim\left(0, \sqrt{2} \sigma_{m}\right) .
\end{array}\right.
$$

Based on positions of three markers, we can obtain two vectors $\hat{\boldsymbol{r}}_{12}^{g}$ and $\hat{\boldsymbol{r}}_{13}^{g}$. Subsequently, the orientation based on optical markers can be estimated as follows. More details are described in Appendix D

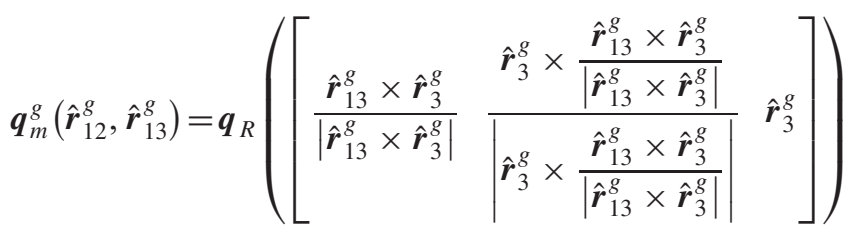

where $\boldsymbol{q}_{R}$ is the transfer function from rotation matrix to quaternion. $\hat{\boldsymbol{r}}_{3}^{g}$ is

$$
\hat{\boldsymbol{r}}_{3}^{g}=\frac{\hat{\boldsymbol{r}}_{12}^{g}}{\left|\hat{\boldsymbol{r}}_{12}^{g}\right|} .
$$

Based on Fig. 1 and (4), we find that the error of relative orientation $\boldsymbol{q}_{m}^{g}\left(\hat{\boldsymbol{r}}_{12}^{g}, \hat{\boldsymbol{r}}_{13}^{g}\right)$ is related to $\alpha, \delta \boldsymbol{p}_{i}$, and $\boldsymbol{p}_{i}$, as described by the following equation:

$$
\boldsymbol{q}_{\mathrm{err}}=\left[\boldsymbol{q}_{m}^{g}\left(\hat{\boldsymbol{r}}_{12}^{g}, \hat{\boldsymbol{r}}_{13}^{g}\right)\right]^{-1} \otimes \boldsymbol{q}_{m}^{g}\left(\boldsymbol{r}_{12}^{g}, \boldsymbol{r}_{13}^{g}\right)
$$

where $\otimes$ represents multiplication between quaternions. The error angle $\left|\boldsymbol{\theta}_{\text {err }}\right|$ is determined by $\boldsymbol{q}_{\mathrm{err}}$. As $\boldsymbol{r}_{12}^{g}$ and $\boldsymbol{r}_{13}^{g}$ are close to $\hat{\boldsymbol{r}}_{12}^{g}$ and $\hat{\boldsymbol{r}}_{13}^{g}$, the rotation error $\boldsymbol{q}_{\mathrm{err}}$ is quite small, and can, therefore, be approximated as

$$
\boldsymbol{q}_{\mathrm{err}} \approx\left[\begin{array}{ll}
1 & \frac{1}{2} \boldsymbol{\theta}_{\mathrm{err}}
\end{array}\right]
$$

In order to investigate the orientation error $\boldsymbol{\theta}_{\text {err }}$ caused by $\alpha$ and $\delta \boldsymbol{p}_{i}$ for varying $\left|\boldsymbol{r}_{i j}\right|$, a simulation-based Monte Carlo analysis was performed. $\delta \boldsymbol{p}_{i}$ was assumed to have a normal distribution with zero mean and standard deviation of $\sigma_{m}$. Fig. 2 shows the simulation results, which indicate that the orientation error increases when $\sigma_{m}$ becomes larger or $\alpha$ smaller. More details can be found in Table II in Appendix A. The error source $\alpha$ is determined by the configuration of markers. Fig. 2(b) indicates that the error induced by $\alpha$ can be minimized by designing $\boldsymbol{r}_{12}$ and $\boldsymbol{r}_{13}$ perpendicular to each other $(\alpha=\pi / 2)$. The error source $\delta \boldsymbol{p}_{i}$, it can be 


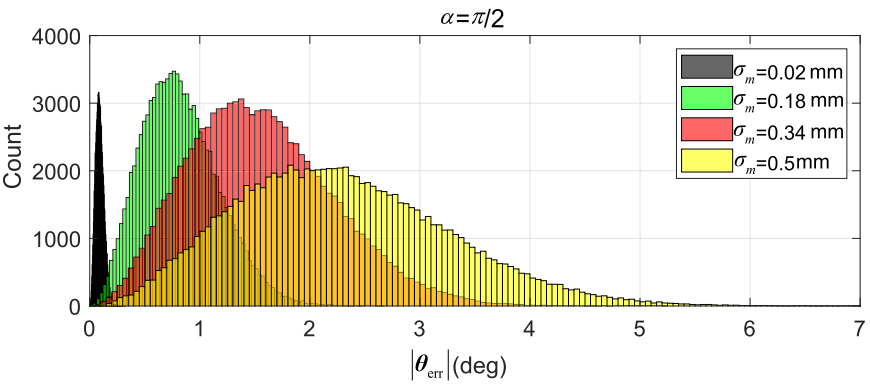

(a)

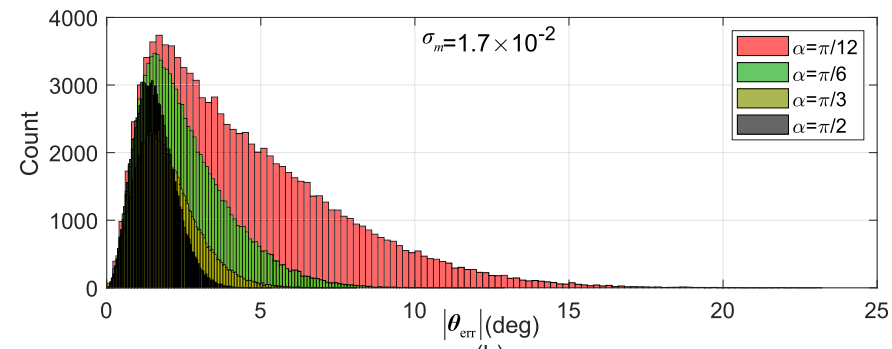

(b)

Fig. 2. Error angle $\left|\boldsymbol{\theta}_{\text {err }}\right|$ for varying angles $\alpha$ and measurement noise $\delta \boldsymbol{p}_{i}$. $\delta \boldsymbol{p}_{i}$ and $\alpha$ are investigated based on Monte Carlo analysis, where $\alpha$ is the angle between two vectors [see Fig. 1 and (1)]. Distance $\left|\boldsymbol{r}_{12}\right|$ and $\left|\boldsymbol{r}_{13}\right|$ are both set as $20 \mathrm{~mm}, \delta \boldsymbol{p}_{i}$ was generated under a normal distribution $\left(0, \sigma_{m}\right), 10^{5}$ particles were used in each simulation. (a) Distribution of $\left|\boldsymbol{\theta}_{\mathrm{err}}\right|$ with different $\sigma_{m}, \alpha$ is set as $\pi / 2$. (b) Distribution of orientation error $\left|\boldsymbol{\theta}_{\mathrm{err}}\right|$ with different $\alpha$.

reduced by reducing the distance between the cameras and the markers or by changing the configuration or number of cameras. Given a certain OTS setup, the influence of $\delta \boldsymbol{p}_{i}$ can be reduced by increasing marker distance $\left|\boldsymbol{r}_{i j}\right|$. However, the space for attachment of markers is quite small for some applications, such as tracking finger movements.

\section{B. Sensor Model}

The gyroscope sensor measures angular velocity in sensor frame, disturbed by various errors, including gain error, nonorthogonality error, and offset error. After sensor calibration, gain, and nonorthogonality error can be eliminated. However, the offset error is a slowly varying variable and has a large accumulating effects when integrating angular velocity to orientation change. Thus, it is estimated as part of the sensor model. The error model of a gyroscope can be simplified as [11]

$$
\boldsymbol{y}_{\mathrm{gyr}}^{s}=\omega^{s}-\boldsymbol{b}^{s}-\boldsymbol{e}^{s}
$$

where $\boldsymbol{y}_{\mathrm{gyr}}^{s}$ is the gyroscope sensor output, $\boldsymbol{\omega}^{s}$ is the corrected angular velocity in sensor frame, $\boldsymbol{b}^{s}$ and $\boldsymbol{e}^{s}$ are corresponding offset error and measurement noise.

\section{Orientation Estimation Based on a Gyroscope}

From a gyroscope, change of orientation over time can be estimated by integrating angular velocity. Since quaternion representation has high efficiency compared with rotation matrices and Euler angles, we use quaternions for describing 3-D orientation and orientation update

$$
\boldsymbol{q}_{g, k+1}^{s}=\boldsymbol{q}_{g, k}^{s} \otimes \delta \boldsymbol{q}_{g, k}^{s}
$$

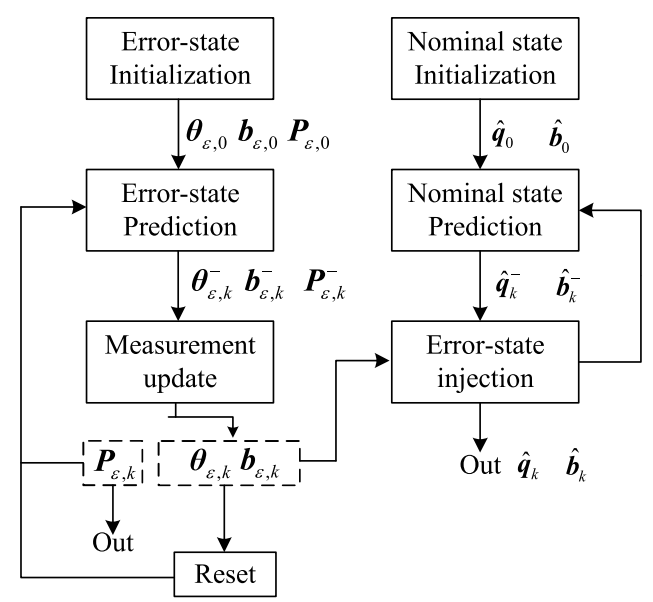

Fig. 3. ESKF design. The ESKF can be divided into two parts. Firstly, update the error-state parameter $\hat{\boldsymbol{\theta}}_{\varepsilon, 0}$ and $\hat{\boldsymbol{b}}_{\varepsilon, 0}$, as shown on the left side. Secondly, injection of the error-state parameter in the nominal-state estimate and, update of the nominal-state parameter $\hat{\boldsymbol{q}}_{k}$ and $\hat{\boldsymbol{b}}_{k}$, as shown on the right side.

$\boldsymbol{q}_{g, k}^{s}$ is the orientation in global frame at time $k$. If the rotation angle in a time step is small, $\delta \boldsymbol{q}_{g, k}^{s}$ can be approximated as

$$
\delta \boldsymbol{q}_{g, k}^{s} \approx\left[\begin{array}{ll}
1 & \frac{1}{2} \delta \boldsymbol{\theta}_{k}^{s}
\end{array}\right]=\left[\begin{array}{ll}
1 & \frac{1}{2} d_{t} \boldsymbol{\omega}_{k}^{s}
\end{array}\right]
$$

where $d_{t}$ is the time interval between time $k$ and $k+1 . \omega_{k}^{s}$ is the angular velocity in sensor frame at time $k$.

\section{Data Fusion Based on ESKF}

We propose an ESKF to fuse gyroscope and OTS-based orientation estimates. This method has an excellent reputation for human body motion tracking using IMMU [25]-[27]. Unlike an extended Kalman filter (EKF) that updates only nominal-state, ESKF updates both the nominal and error-state estimates. The true-state is estimated by the sum of nominal and error-states. The nominal and error-states are considered as larger and small state components. The nominal-state does not consider noise terms while the error-state estimate incorporates noise and perturbations. The small error state enables the formulation of a linearized error state model and easier computation of Jacobians [28]. The applied ESKF design is shown in Fig. 3.

The orientation and offset of gyroscope are included in the state vector. The true-state, nominal-state, and error-state are defined as

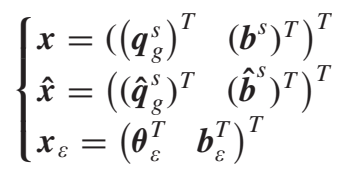

where ${ }^{\wedge}$ represents nominal-state estimate, $\varepsilon$ represents errorstate. $\boldsymbol{q}_{g}^{s}$ and $\boldsymbol{b}^{s}$ are quaternion-based orientation and offset of the gyroscope. $\boldsymbol{\theta}_{\varepsilon}$ and $\boldsymbol{b}_{\varepsilon}$ are error-states related to $\boldsymbol{q}_{g}^{s}$ and $\boldsymbol{b}^{s}$. $\boldsymbol{q}_{g}^{s}, \hat{\boldsymbol{q}}_{g}^{s}, \boldsymbol{b}^{s}$, and $\hat{\boldsymbol{b}}^{s}$ are abbreviated as $\boldsymbol{q}, \hat{\boldsymbol{q}}, \boldsymbol{b}$, and $\hat{\boldsymbol{b}}$ below.

As shown in Fig. 3, the update of the orientation $\boldsymbol{q}_{k}$ and gyroscope offset $\boldsymbol{b}_{k}$ includes the update of the error-state and nominal-state. The process can be divided into four steps: the first step is to initialize the orientation and gyroscope offset, including the error-state and nominal-state. The second step is 
to predict the error-state and nominal-state for the orientation and gyroscope offset. The prediction for the nominal-state is mainly deduced by the integration of angular velocity exploiting (9) and (10). The prediction for the error-states is mainly deduced by the differential of the relation between the true-state and nominal-state [see (23) and (24)]. The third step is to update the error-state based on measurement data. The relation between measurement data and error-states can be obtained from the described process model that describes the relationship between the measurement data and true-state. The fourth step is to obtain a prediction of the true-state based on the prediction of the nominal-state and updated error-state. More details are as follows.

1) Initialization: The initial value of the nominal-state $\hat{\boldsymbol{q}}_{0}$ is obtained from the OTS. The initial values for error-states $\hat{\boldsymbol{b}}_{0}$, $\boldsymbol{\theta}_{\varepsilon, 0}$, and $\boldsymbol{b}_{\varepsilon, 0}$ are set to $\mathbf{0}$. Before every prediction and update, we obtain the angular velocity $\boldsymbol{y}_{\mathrm{gyr}}^{s}$ from the gyroscope, and know the nominal-state estimate $\hat{\boldsymbol{x}}_{k-1}$ at time $k-1$, and the covariance matrix $\boldsymbol{P}_{\varepsilon, k-1}$ belonging to estimated error-state $\boldsymbol{x}_{\varepsilon, k-1}$.

2) Prediction: The purpose of this step is to predict the nominal-state $\hat{\boldsymbol{x}}_{k}^{-}$, error-state $\boldsymbol{x}_{\varepsilon, k}^{-}$and the corresponding covariance matrix $\boldsymbol{P}_{\varepsilon, k}^{-}$, where ${ }^{-}$denotes the prior estimation.

a) Nominal-state prediction: The offset error of the gyroscope $\boldsymbol{b}$ is modeled as a first order Markov process driven by white noise $\boldsymbol{n}_{b, k}$

$$
\boldsymbol{b}_{k}=\boldsymbol{b}_{k-1}+\boldsymbol{n}_{b, k} .
$$

For prediction of $\boldsymbol{b}$, we assume

$$
\hat{\boldsymbol{b}}_{k}^{-}=\hat{\boldsymbol{b}}_{k-1}
$$

The prediction of orientation based on the gyroscope is performed by using (9) and (10)

$$
\hat{\boldsymbol{q}}_{k}^{-}=\left(\boldsymbol{I}_{4 \times 4}+\frac{1}{2} \boldsymbol{\Omega}_{k-1} d_{t}\right) \hat{\boldsymbol{q}}_{k-1}
$$

where $\boldsymbol{\Omega}_{k-1}$ is

$$
\boldsymbol{\Omega}_{k-1}=\left[\begin{array}{cccc}
0 & -\hat{\omega}_{x}^{s} & -\hat{\omega}_{y}^{s} & -\hat{\omega}_{z}^{s} \\
\hat{\omega}_{x}^{s} & 0 & \hat{\omega}_{z}^{s} & -\hat{\omega}_{y}^{s} \\
\hat{\omega}_{y}^{s} & -\hat{\omega}_{z}^{s} & 0 & \hat{\omega}_{x}^{s} \\
\hat{\omega}_{z}^{s} & \hat{\omega}_{y}^{s} & -\hat{\omega}_{x}^{s} & 0
\end{array}\right]_{k-1}
$$

and $\hat{\omega}_{x}^{s}, \hat{\omega}_{y}^{s}, \hat{\omega}_{z}^{s}$ are components of nominal angular velocity $\hat{\omega}_{k-1}^{s}$. $\hat{\omega}_{k}^{s}$ can be obtained according to (8)

$$
\hat{\boldsymbol{\omega}}_{k}^{s}=\boldsymbol{y}_{\mathrm{gyr}, k}^{s}+\hat{\boldsymbol{b}}_{k} \text {. }
$$

The prediction for nominal-state $\hat{\boldsymbol{x}}_{k}$ is given by

$$
\hat{\boldsymbol{x}}_{k}^{-}=\boldsymbol{F}_{k} \hat{\boldsymbol{x}}_{k-1}
$$

where the transition matrix $\boldsymbol{F}_{k}$ is

$$
\boldsymbol{F}_{k}=\left[\begin{array}{cc}
\left(\boldsymbol{I}_{4 \times 4}+\frac{d_{t}}{2} \boldsymbol{\Omega}_{k}\right) & \mathbf{0}_{4 \times 3} \\
\mathbf{0}_{3 \times 4} & \boldsymbol{I}_{3 \times 3}
\end{array}\right] .
$$

b) Error-state prediction: The error-state of the gyroscope offset $\boldsymbol{b}_{\varepsilon, k}$ is defined as the difference between the estimated and true value of $\boldsymbol{b}_{k}$

$$
\boldsymbol{b}_{\varepsilon, k}=\hat{\boldsymbol{b}}_{k}-\boldsymbol{b}_{k} \text {. }
$$

If we substitute (12) and (13) in (19), we find

$$
\boldsymbol{b}_{\varepsilon, k}=\hat{\boldsymbol{b}}_{k}-\boldsymbol{b}_{k}=\hat{\boldsymbol{b}}_{k-1}-\left(\boldsymbol{b}_{k-1}+\boldsymbol{n}_{b, k}\right)=\boldsymbol{b}_{\varepsilon, k-1}-\boldsymbol{n}_{b, k} \text {. }
$$

The prediction for the error-state $\boldsymbol{b}_{\varepsilon, k}$ is

$$
\boldsymbol{b}_{\varepsilon, k}^{-}=\boldsymbol{b}_{\varepsilon, k-1} \text {. }
$$

For a small orientation error denoted $\boldsymbol{\theta}_{\varepsilon}$, the corresponding quaternion-based orientation error can be approximated as

$$
\boldsymbol{q}_{\varepsilon} \approx\left[\begin{array}{ll}
1 & \frac{1}{2} \boldsymbol{\theta}_{\varepsilon}
\end{array}\right] \text {. }
$$

The true quaternion can be expressed as

$$
\boldsymbol{q}=\hat{\boldsymbol{q}} \otimes \boldsymbol{q}_{\varepsilon} .
$$

If we differentiate (23) on both sides, we obtain the following equation, more details can be found in the work of Schepers et al. [29] and Weenk et al. [30]:

$$
\dot{\boldsymbol{\theta}}_{\varepsilon}=-\left\lfloor\hat{\boldsymbol{\omega}}^{s}\right\rfloor_{\times} \boldsymbol{\theta}_{\varepsilon}-\boldsymbol{\omega}_{\varepsilon}^{s}
$$

where $\hat{\omega}^{s}$ can be obtained from (16). $\rfloor_{\times}$denotes a skewsymmetric matrix. More details can be found in Appendix C. $\boldsymbol{\omega}_{\varepsilon}^{s}$ is the difference between the estimated and true angular velocity

$$
\begin{aligned}
\boldsymbol{\omega}_{\varepsilon}^{s} & =\hat{\boldsymbol{\omega}}^{s}-\boldsymbol{\omega}^{s} \\
& =\boldsymbol{y}_{\mathrm{gyr}}^{s}+\hat{\boldsymbol{b}}-\left(\boldsymbol{y}_{\mathrm{gyr}}^{s}+\boldsymbol{b}+\boldsymbol{e}^{s}\right) \\
& =\boldsymbol{b}_{\varepsilon}-\boldsymbol{e}^{s} .
\end{aligned}
$$

By combining (24) and (25), the orientation error can be expressed as

$$
\boldsymbol{\theta}_{\varepsilon, k}=\left(\boldsymbol{I}_{3 \times 3}-\left\lfloor\hat{\boldsymbol{\omega}}_{k-1}^{s}\right\rfloor_{\times} d_{t}\right) \boldsymbol{\theta}_{\varepsilon, k-1}-\left(\boldsymbol{b}_{\varepsilon, k-1}-\boldsymbol{e}^{s}\right) d_{t} .
$$

Based on (26), the prediction for orientation error $\boldsymbol{\theta}_{\varepsilon, k}$ is updated as

$$
\boldsymbol{\theta}_{\varepsilon, k}^{-}=\left(\boldsymbol{I}_{3 \times 3}-\left\lfloor\hat{\boldsymbol{\omega}}_{k-1}^{s}\right\rfloor_{\times} d_{t}\right) \boldsymbol{\theta}_{\varepsilon, k-1}-\boldsymbol{b}_{\varepsilon, k-1} d_{t} .
$$

From (20) and (26), we can obtain the process model for the error-state $\boldsymbol{x}_{\varepsilon, k}$

$$
\boldsymbol{x}_{\varepsilon, k}=\boldsymbol{F}_{\varepsilon, k} \boldsymbol{x}_{\varepsilon, k-1}+\boldsymbol{n}_{\boldsymbol{x}_{k}}
$$

where $\boldsymbol{n}_{\boldsymbol{x}_{k}}$ is the noise of the process model. Transition matrix $\boldsymbol{F}_{\varepsilon, k}$ can be expressed as

$$
\boldsymbol{F}_{\varepsilon, k}=\left[\begin{array}{cc}
\frac{\partial\left(\boldsymbol{\theta}_{\varepsilon, k}\right)}{\partial\left(\boldsymbol{\theta}_{\varepsilon, k-1}\right)} & \frac{\partial\left(\boldsymbol{\theta}_{\varepsilon, k}\right)}{\partial \boldsymbol{b}_{\varepsilon, k-1}} \\
\mathbf{0}_{3 \times 3} & \boldsymbol{I}_{3 \times 3}
\end{array}\right] .
$$

Details of the elements in the first row can be found in Appendix C. The final prediction formula for error-state $\boldsymbol{x}_{\varepsilon, k}$ is

$$
\boldsymbol{x}_{\varepsilon, k}^{-}=\boldsymbol{F}_{\varepsilon, k} \boldsymbol{x}_{\varepsilon, k-1} \text {. }
$$

The covariance matrix $\boldsymbol{P}$ is updated using

$$
\boldsymbol{P}_{\varepsilon, k}^{-}=\boldsymbol{F}_{\varepsilon, k} \boldsymbol{P}_{\varepsilon, k-1} \boldsymbol{F}_{\varepsilon, k}^{T}+\boldsymbol{Q}_{\varepsilon}
$$

where $\boldsymbol{Q}_{\varepsilon}$ is the noise covariance matrix of $\boldsymbol{n}_{\boldsymbol{x}_{k}}$. 
3) Measurement Update: From the OTS, we obtain the orientation from marker frame to global frame $\boldsymbol{q}_{m}^{g}$ based on (4). Thus, the orientation from global frame to marker frame $\boldsymbol{q}_{g}^{m}$ is known. $\boldsymbol{y}_{k}$ can be expressed as

$$
\boldsymbol{y}_{k}=\left(\boldsymbol{q}_{g}^{m}\right)_{k}+\boldsymbol{n}_{\boldsymbol{y}_{k}}=\left(\boldsymbol{q}_{s}^{m}\right)_{k} \otimes\left(\boldsymbol{q}_{g}^{s}\right)_{k}+\boldsymbol{n}_{\boldsymbol{y}_{k}}
$$

where $\boldsymbol{q}_{s}^{m}$ is the orientation from sensor frame to marker frame, which can be estimated from calibration movements prior the experiment. Equation (32) can be simplified as

$$
\hat{\boldsymbol{y}}_{k}=\left(\boldsymbol{q}_{g}^{s}\right)_{k}+\boldsymbol{n}_{\boldsymbol{y}_{k}}
$$

where $\hat{\boldsymbol{y}}_{k}$ is the estimation of $\boldsymbol{y}_{k}$ from measurement data

$$
\hat{\boldsymbol{y}}_{k}=\left(\boldsymbol{q}_{s}^{m}\right)_{k}^{-1} \otimes \boldsymbol{y}_{k}
$$

Based on (22) and (23), (33) can be expressed as

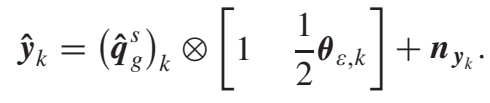

Based on (35), the measurement model can be updated as

$$
\hat{\boldsymbol{y}}_{k}=\boldsymbol{H}_{\varepsilon, k} \boldsymbol{x}_{\varepsilon, k}+\boldsymbol{n}_{\boldsymbol{y}_{k}}
$$

where $\boldsymbol{H}_{\varepsilon, k}$ is the Jacobian matrix of $\hat{\boldsymbol{y}}_{k}$. Details can be found in the Appendix $\mathrm{C}$

$$
\boldsymbol{H}_{\varepsilon, k}=\left[\begin{array}{ll}
\frac{\partial \hat{\boldsymbol{y}}_{k}}{\partial\left(\boldsymbol{\theta}_{\varepsilon, k}\right)} & \frac{\partial \hat{\boldsymbol{y}}_{k}}{\partial\left(\boldsymbol{b}_{\varepsilon, k}\right)}
\end{array}\right] .
$$

Subsequently, we can update the Kalman gain $\boldsymbol{K}_{\varepsilon, k}$

$$
\boldsymbol{K}_{\varepsilon, k}=\boldsymbol{P}_{\varepsilon, k}^{-} \boldsymbol{H}_{\varepsilon, k}^{T}\left(\boldsymbol{H}_{\varepsilon, k} \boldsymbol{P}_{\varepsilon, k}^{-} \boldsymbol{H}_{\varepsilon, k}^{T}+\boldsymbol{R}_{\varepsilon}\right)^{-1}
$$

where $\boldsymbol{R}_{\varepsilon}$ is the measurement noise variance related to error $\boldsymbol{n}_{\boldsymbol{y}_{k}}$. The prior error-state $\boldsymbol{x}_{\varepsilon, k}^{-}$is updated based on the Kalman gain above

$$
\boldsymbol{x}_{\varepsilon, k}=\boldsymbol{x}_{\varepsilon, k}^{-}+\boldsymbol{K}_{\varepsilon, k}\left(\hat{\boldsymbol{y}}_{k}-\boldsymbol{H}_{\varepsilon, k} \boldsymbol{x}_{\varepsilon, k}^{-}\right) .
$$

The error covariance matrix $\boldsymbol{P}_{\varepsilon, k}^{-}$is updated using

$$
\boldsymbol{P}_{\varepsilon, k}=\left(\boldsymbol{I}-\boldsymbol{K}_{\varepsilon, k} \boldsymbol{H}_{\varepsilon, k}\right) \boldsymbol{P}_{\varepsilon, k}^{-} .
$$

4) Injection of the Observed Error Into the Nominal-State: Based on (19), the two components of $\boldsymbol{x}_{\varepsilon, k}$ are $\boldsymbol{b}_{\varepsilon, k}$ and $\boldsymbol{\theta}_{\varepsilon, k}$. $\boldsymbol{b}_{k}$ can be updated as

$$
\hat{\boldsymbol{b}}_{k}=\hat{\boldsymbol{b}}_{k}^{-}-\boldsymbol{b}_{\varepsilon, k}
$$

The orientation $\hat{\boldsymbol{q}}_{k}$ from (24) can be corrected from the $\boldsymbol{\theta}_{\varepsilon, k}$

$$
\hat{\boldsymbol{q}}_{k}=\hat{\boldsymbol{q}}_{k}^{-} \otimes\left[1 \quad \frac{1}{2} \boldsymbol{\theta}_{\varepsilon, k}\right] .
$$

After the nominal-state is corrected, the error-state is reset for the next iteration.

\section{E. Smoothing}

In many biomedical applications, the full measurement sequence is available and the data can be analyzed offline [31]-[33]. Smoothing technique can be used to improve the accuracy of state estimation. For the ESKF mentioned above, the state vector $\boldsymbol{x}_{k}$ is estimated based on the measurement from $\boldsymbol{y}_{1}$ to $\boldsymbol{y}_{k}$, satisfying the causality requirement for realtime processing. With the offline smoothing technique, $\boldsymbol{x}_{k}$ can be estimated noncausally with all the measurements from $\boldsymbol{y}_{1}$ to $\boldsymbol{y}_{N}$. More importantly, the smoothing technique can improve the estimation during the line of sight occlusion. Since it can estimate the orientation by integrating angular velocity both forward and backward in time during the occlusion.

Among all smoothers, Rauch-Tung-Striebel (RTS) smoother exploits fixed-interval Kalman smoothing technique [34]-[36] and is well known for its efficiency. Therefore, we chose this smoother for improving the estimation accuracy under offline condition. The implementation of RTS can be divided into two steps: The first step is to apply the ESKF described above and save the estimates for $\hat{\boldsymbol{q}}_{k}, \hat{\boldsymbol{b}}_{k}$, $\boldsymbol{x}_{\varepsilon, k}, \boldsymbol{x}_{\varepsilon, k}^{-}, \boldsymbol{P}_{\varepsilon, k}$, and $\boldsymbol{P}_{\varepsilon, k}^{-}$. The second step is to perform a backward EKF which computes the smoothing state vector with the parameter saved in the prior ESKF. The gain $\boldsymbol{A}_{\varepsilon, k}$ is calculated as

$$
\boldsymbol{A}_{\varepsilon, k}=\boldsymbol{P}_{\varepsilon, k} \boldsymbol{F}_{\varepsilon, k}^{T}\left(\boldsymbol{P}_{\varepsilon, k+1}^{-}\right)^{-1}
$$

Subsequently, the state vector $\boldsymbol{x}_{\varepsilon, k}$ is updated based on $\boldsymbol{A}_{\varepsilon, k}$

$$
\boldsymbol{x}_{s \mid \varepsilon, k}=\boldsymbol{x}_{\varepsilon, k}+\boldsymbol{A}_{\varepsilon, k}\left(\boldsymbol{x}_{s \mid \varepsilon, k+1}-\boldsymbol{x}_{\varepsilon, k}^{-}\right) .
$$

Finally, the covariance matrix $\boldsymbol{P}_{\varepsilon, k}$ is updated as

$$
\boldsymbol{P}_{s \mid \varepsilon, k}=\boldsymbol{P}_{\varepsilon, k}+\boldsymbol{A}_{\varepsilon, k}\left(\boldsymbol{P}_{s \mid \varepsilon, k+1}-\boldsymbol{P}_{\varepsilon, k+1}^{-}\right)
$$

where $\boldsymbol{x}_{s \mid \varepsilon, k}$ and $\boldsymbol{P}_{s \mid \varepsilon, k}$ are estimated state vector and variance using the smoothing technique. The initial values are set as

$$
\boldsymbol{x}_{s \mid \varepsilon, N}=\boldsymbol{x}_{\varepsilon, N} ; \quad \boldsymbol{P}_{s \mid \varepsilon, N}=\boldsymbol{P}_{\varepsilon, N} .
$$

Based on (19) and (23), the offset of the gyroscope $\hat{\boldsymbol{b}}_{k}$ is updated as

$$
\hat{\boldsymbol{b}}_{k}=\hat{\boldsymbol{b}}_{k}^{-}-\boldsymbol{b}_{s \mid \varepsilon, k} .
$$

The orientation $\hat{\boldsymbol{q}}_{k}$ is updated as

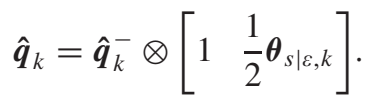

\section{EXPERIMENTS}

To validate the proposed algorithm, two experiments were performed. The first experiment was to evaluate the performance of the algorithm during 3-D static and dynamic movements. The measurement unit including a cluster of markers and a gyroscope was placed statically and rotated in 3-D space with yaw, pitch, and roll rotations. The second experiment was to evaluate its performance during a biomedical application, being the estimation of the relative orientation between the hand and the index finger during a flexion and extension movement. 


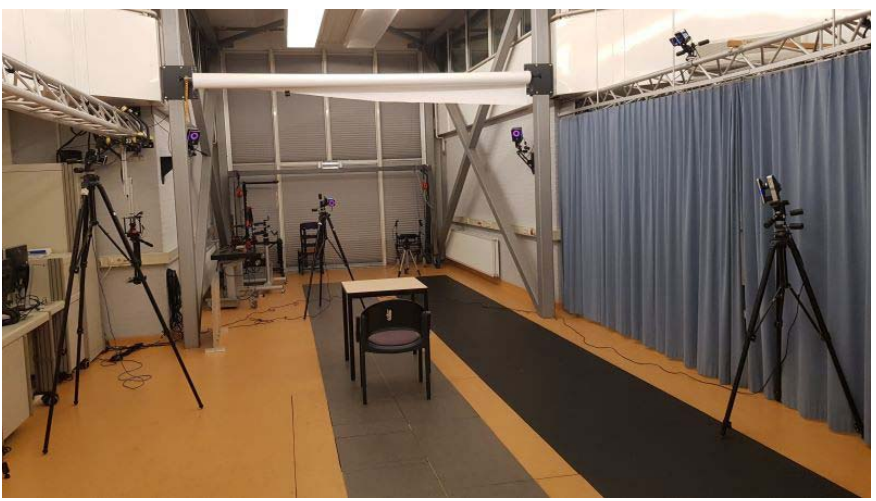

Fig. 4. Vicon system with eight cameras. A table in the middle of cameras was used as a support during the static experiment. All the data were preprocessed with the Vicon Nexus 2.10.2 software.

\section{A. Experiment Setups}

We used 3-D rate gyroscopes included in the MPU9250 (InveSense) in our experiment. The sampling frequency of the gyroscope was $200 \mathrm{~Hz}$. The data were transmitted to the PC through a USB connection. The Vicon system with eight cameras, as shown in Fig. 4, was chosen as the OTS system. The sampling frequency was $100 \mathrm{~Hz}$.

In the first experiment, the cluster of markers formed an isosceles right triangle $p_{1} p_{2} p_{3}$, which is attached above the surface of the gyroscope, as shown in Fig. 5. The distances between markers depend on applications. It will, for example, be different when comparing human leg tracking with fingertip tracking. In order to evaluate the performance of the proposed method for different distances between markers, the leg $r_{12}$ was set to three different values: 13,18 , and $24 \mathrm{~mm}$. Another cluster of markers forms a second isosceles right triangle $P_{1} P_{2} P_{3}$. The leg $R_{12}$ was $100 \mathrm{~mm}$. The orientation estimate based on $P_{1} P_{2} P_{3}$ is much more accurate than the orientation estimate based on $p_{1} p_{2} p_{3}$, and was therefore used as an orientation reference.

In the second experiment, two measurement units were attached to the dorsal side of the hand and the index fingertip, as shown in Fig. 6. No orientation reference was used in this experiment.

\section{B. Synchronization}

The gyroscope and OTS. Both systems were independent and sampled individually. Therefore, they needed to be synchronized before data fusion. We obtained an estimate of the modulus of the angular velocity from the OTS system in the following manner: First, we determined the quaternion-based orientation from the markers using (4)

$$
\tilde{\boldsymbol{q}}_{g, k}^{m}=\left(\boldsymbol{q}_{m, k}^{g}\right)^{-1} \text {. }
$$

Using (9), we derived

$$
\delta \tilde{\boldsymbol{q}}_{g, k}^{m}=\left(\tilde{\boldsymbol{q}}_{g, k}^{m}\right)^{-1} \otimes \tilde{\boldsymbol{q}}_{g, k+1}^{m} .
$$

Subsequently, we estimated the angular velocity $\tilde{\boldsymbol{\omega}}_{k}^{m}$ using the following relation:

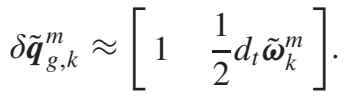

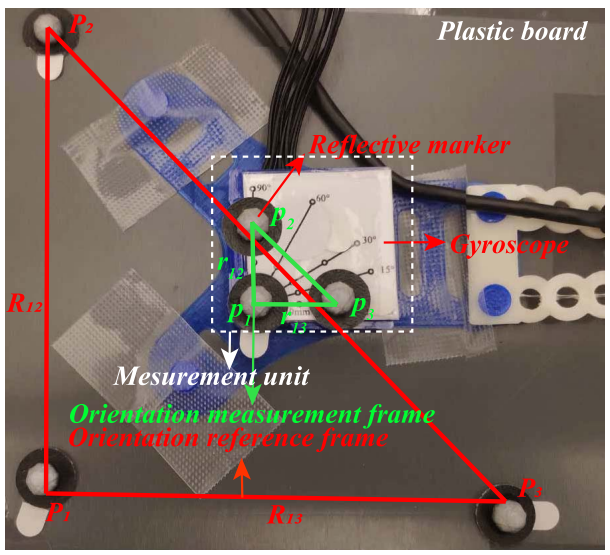

Fig. 5. Markers and the gyroscope setup. One cluster of markers was attached on top of a gyroscope. It formed an isosceles right triangle $p_{1} p_{2} p_{3}$. The other cluster of markers form an isosceles right triangle $P_{1} P_{2} P_{3}$. The leg $R_{12}$ of the second marker configuration was much longer than $r_{12}$ of the first configuration. Thus, the orientation from $P_{1} P_{2} P_{3}$ is more accurate and was therefore used as an orientation reference. The length of $r_{12}$ was varied, the length of $R_{12}$ was approximately $100 \mathrm{~mm}$.

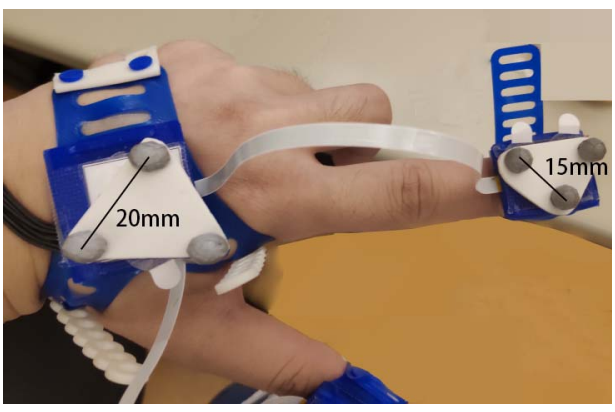

Fig. 6. Measurement units used to estimate relative orientation between hand and index finger. Every measurement unit includes a gyroscope with a cluster of markers attached. One unit was attached to the dorsal side of the hand, the other to the tip of the index finger. The shorter legs of the isosceles right triangle formed by markers were 20 and $15 \mathrm{~mm}$ on dorsal side of the hand and index finger respectively.

From the gyroscope, we obtained the measured angular velocity $\boldsymbol{y}_{\mathrm{gyr}, k}^{s}$. Using (8), we estimate $\tilde{\boldsymbol{\omega}}_{k}^{m}$ from $\boldsymbol{y}_{\mathrm{gyr}, k}^{s}$, taking into account offset $\boldsymbol{b}$, noise $\boldsymbol{e}^{m}$ and sensor to maker frame rotation $\boldsymbol{q}_{s, k}^{m}$. However, during the rotation, $\boldsymbol{b}$ and noise $\boldsymbol{e}^{m}$ are much smaller than $\boldsymbol{y}_{\mathrm{gyr}, k}^{s}$. The moduli of angular velocities from the OTS and the gyroscope are therefore assumed to be approximately the same, that is,

$$
\left(\tilde{\boldsymbol{\omega}}_{k}^{m}\right)^{T} \tilde{\boldsymbol{\omega}}_{k}^{m} \approx\left(\boldsymbol{y}_{\mathrm{gyr}, k}^{s}\right)^{T} \boldsymbol{y}_{\mathrm{gyr}, k}^{s}
$$

The synchronization was done by maximizing the correlation between $\left|\tilde{\boldsymbol{\omega}}_{k}^{m}\right|$ and $\left|\boldsymbol{y}_{\mathrm{gyr}, k}^{s}\right|$.

\section{Alignment}

1) Alignment Between Markers and the Gyroscope in Both Experiments: In addition to synchronization, the alignment between the gyroscope and optical markers is vital for data fusion, since it is required by comparing the angular velocity calculated by the markers and measured by the gyroscope.

According to (8), $\boldsymbol{q}_{s}^{m}$ can be obtained with

$$
\operatorname{argmin}_{\boldsymbol{q}_{s}^{m}}\left\|\overline{\boldsymbol{\omega}}^{m}-\boldsymbol{q}_{s}^{m} \otimes\left(\overline{\boldsymbol{y}}_{\mathrm{gyr}}^{s}-\boldsymbol{b}^{s}\right)\right\|_{2}^{2} .
$$

The result of alignment between the gyroscope and OTS is described in Appendix B. 

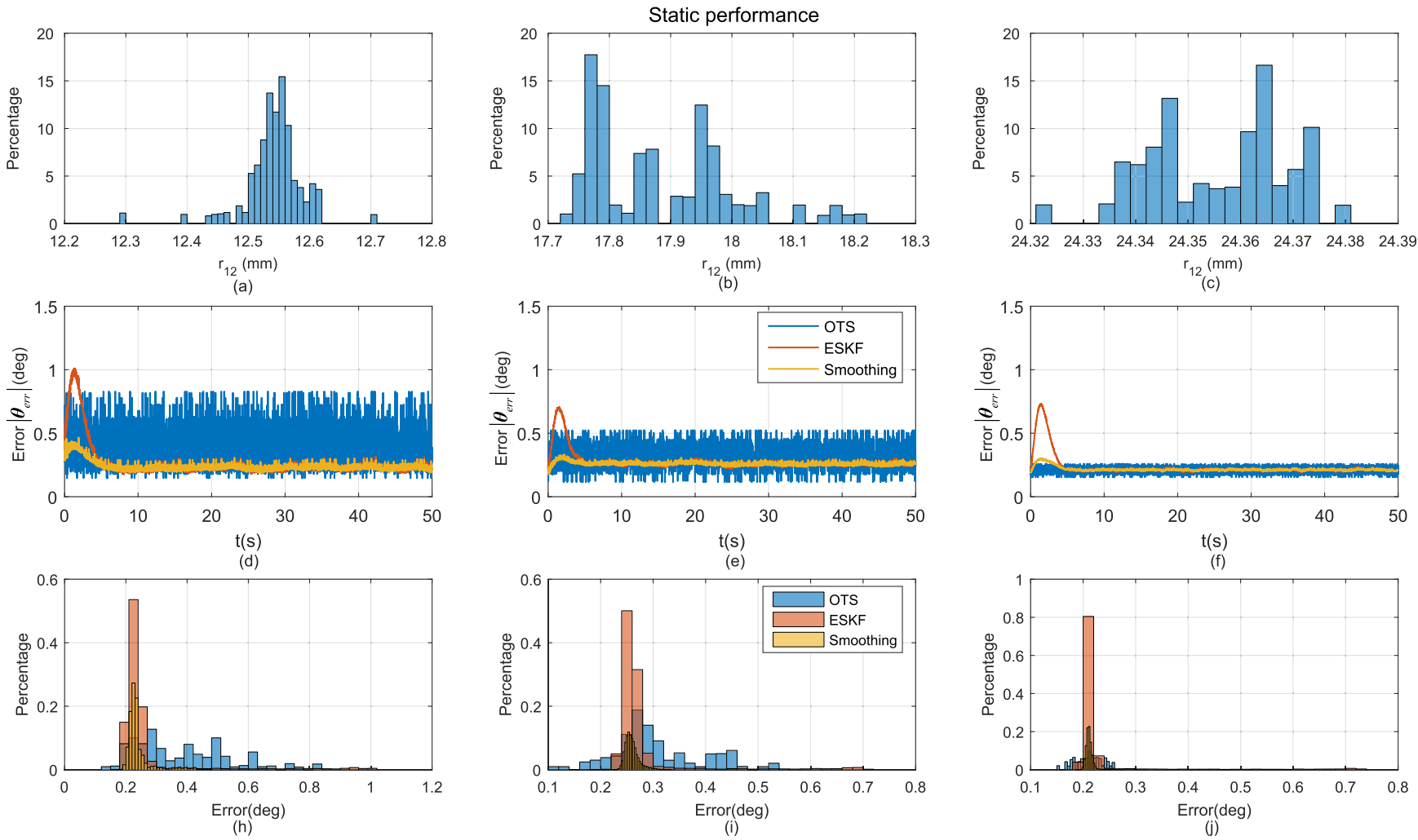

Fig. 7. Orientation estimation error $\left|\boldsymbol{\theta}_{\text {err }}\right|$ in a static orientation with different marker distances. (a)-(c) Marker distances measured using OTS. (d)-(f) Estimated orientation error with different methods for the three classes of marker distances shown in (a)-(c) respectively. (h)-(j) Corresponding error distributions.

2) Alignment Between Two Clusters of Markers in Experiment 1: Before the cluster $P_{1} P_{2} P_{3}$ can be used as reference for $p_{1} p_{2} p_{3}$, it is essential to align them. The relative orientation between the two clusters can be estimated from the orientation of each cluster with respect to the global frame according to (4) when the object with both marker clusters is held in a static posture. The error angle $\boldsymbol{\theta}_{\text {err }}$ showed in Figs. 7 and 8 is determined in (6) and (7).

\section{Experiment Procedures}

1) Experiment 1-Orientation Estimation in 3-D Space: Two cluster markers and an IMU (MPU925) were attached to a rigid plate, as shown in Fig. 5. The plate held still for $60 \mathrm{~s}$ in one orientation and was subsequently rotated randomly in 3-D space for $60 \mathrm{~s}$. Subsequently, the length of $r_{12}$ was changed from 13 to $17 \mathrm{~mm}$ and $24 \mathrm{~mm}$, and the static and dynamic movements were repeated.

The setup of markers and cameras was designed such that all markers were always visible by enough cameras. The line of sight occlusion was implemented in the analysis by leaving out the position of one or several of the markers of cluster $p_{1} p_{2} p_{3}$ during a certain time period. During the line-of-sight occlusion, the positions of the reference set $P_{1} P_{2} P_{3}$ were visible, and therefore the reference orientation, was available.

2) Experiment 2-Relative Orientation Estimation Between Hand and Fingers: Two marker clusters and gyroscopes were attached on the dorsal side of the hand and index fingertip, as shown in Fig. 6. First, the sensor to segment calibration was performed, including the sensor to hand and the sensor to index fingertip calibration.
1) The hand was placed on a horizontally flat surface, the back of the palm pointing upward.

2) The hand was placed against a vertically flat surface.

3) The hand was raised and the index finger was repeatedly flexed and extended.

After the above procedure, the sensor to segment calibration was done by exploiting that a static accelerometer only measures gravitational acceleration and a gyroscope measures angular velocity. More details about the sensor to segment calibration can be found in [11].

Finally, flexion and extension movements were performed with the index finger, approximately once per second, while not rotating nor displacing the hand. The rotation angle for the index finger was approximately $150^{\circ}$. The orientation of the whole hand was subsequently changed and the flexion and extension movements repeated.

\section{RESUlTS}

\section{A. Experiment 1-Orientation Estimation in 3-D Space}

1) Static Performance: The results of the static trials are shown in Fig. 7. Fig. 7(a)-(c) show the marker distances $r_{12}$, while (d) to (f) show the corresponding orientation errors, and (h) to (j) are the corresponding error distributions in (d) to (f). When marker distance $r_{12}$ increased, the corresponding orientation estimation error became smaller. Initial peaks in Fig. 7(d)-(f) are the result of "switch-on transient behavior" due to the initial value of the error covariance matrix $\boldsymbol{P}_{\varepsilon, k}$. When applying the ESKF separately or in combination with the smoothing method, the estimation errors reduced extensively. The estimation errors when the ESKF was applied 


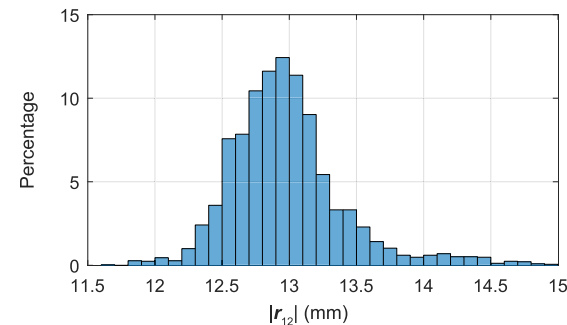

(a)

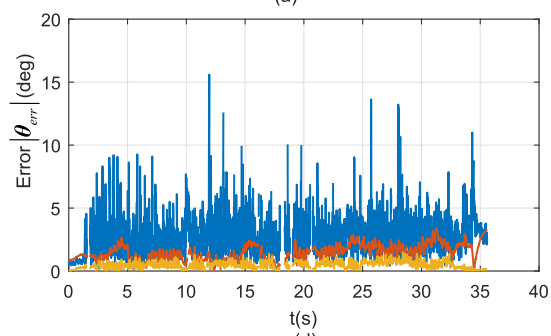

(d)

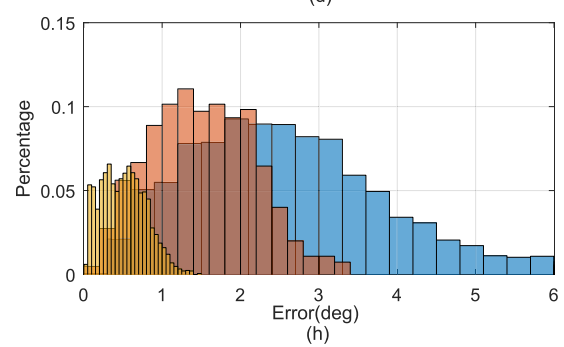

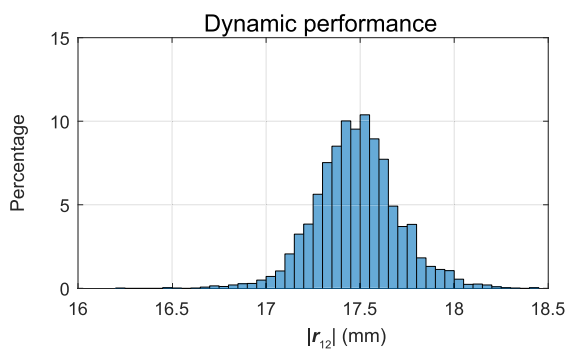

(b)

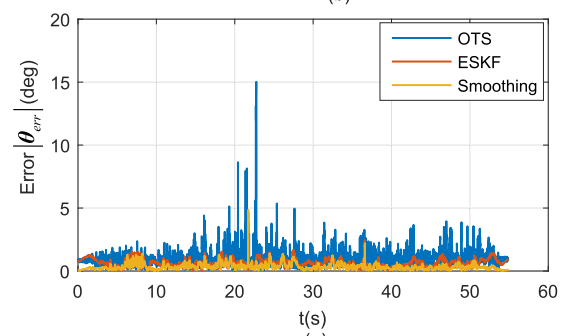

(e)

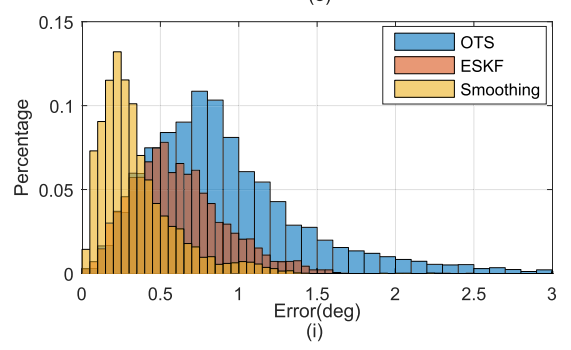

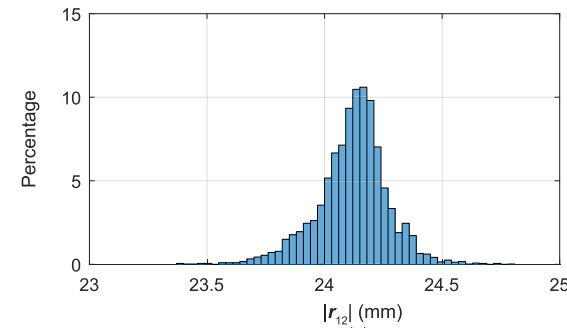

(c)
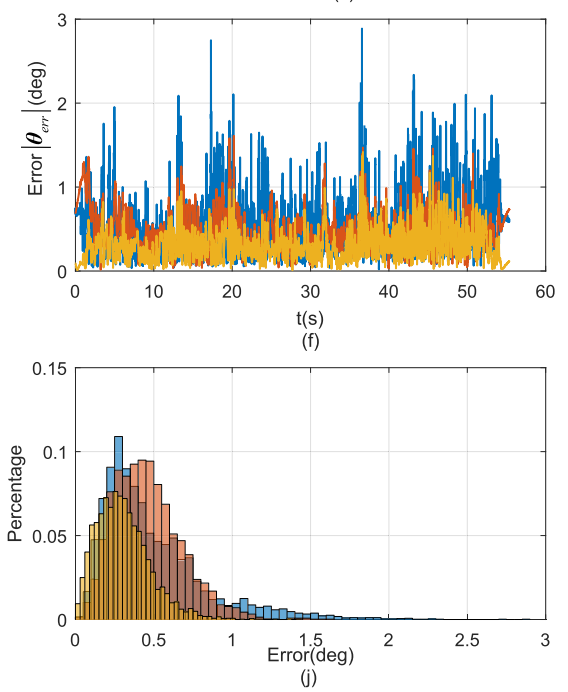

Fig. 8. Orientation estimation error $\left|\boldsymbol{\theta}_{\text {err }}\right|$ during 3-D movements for different marker distance. (a)-(c) Marker distance measured using OTS. (d)-(f) Estimated orientation error with different methods relative to (a)-(c) respectively. (h)-(j) Corresponding error distributions. The orientation error is defined in (7).

separately or in combination with the smoother did not differ significantly. The detailed orientation errors are specified in Table I.

2) Dynamic Performance: The results during dynamic trials are shown in Fig. 8. These results demonstrate two advantages of the proposed fusion method when compared with applying only the OTS: both accuracy and orientation estimation during the line of sight occlusion improved.

3) Accuracy Improvement: Fig. 8(a)-(c) shows the measured marker distances $r_{12}$ for the three marker configurations that were evaluated, while (d)-(f) show the corresponding orientation errors, and (h)-(j) are the corresponding error distributions under the condition that no line-of-sight occlusion was implemented. When marker distance $r_{12}$ increased, the corresponding orientation error appeared to become smaller. Furthermore, the orientation error was smaller after fusion with the gyroscope using an ESKF and additional smoothing. The smoothing technique improved the result of ESKF. The detailed values are specified in Table I.

4) Data Filling During Line-of-Sight Occlusion: The optical data between $20 \mathrm{~s}$ and $40 \mathrm{~s}$ was assigned as unavailable (Fig. 9). During this period, the orientation was filled with gyroscope data automatically. As shown in Fig. 9, The orientation error increased to $4.8^{\circ}$ in $20 \mathrm{~s}$ by only exploiting the gyroscope, which was reduced to $2.5^{\circ}$ by additionally using the smoothing technique.
TABLE I

ORIENTATION ERROR WITH OTS AND ESKF FOR DIFFERENT MARKER DisTANCES

\begin{tabular}{ccccc}
\hline Items & & $\begin{array}{l}r_{12}= \\
13 \mathrm{~mm}\end{array}$ & $\begin{array}{c}r_{12}= \\
18 \mathrm{~mm}\end{array}$ & $\begin{array}{l}r_{12}= \\
24 \mathrm{~mm}\end{array}$ \\
\hline \multirow{2}{*}{ OTS (deg) } & static & $0.39 \pm 0.16$ & $0.31 \pm 0.08$ & $0.21 \pm 0.03$ \\
& dynamic & $2.75 \pm 1.56$ & $0.96 \pm 0.74$ & $0.49 \pm 0.34$ \\
ESKF (deg) & static & $0.23 \pm 0.02$ & $0.26 \pm 0.01$ & $0.21 \pm 0.00$ \\
& dynamic & $1.50 \pm 0.62$ & $0.54 \pm 0.26$ & $0.43 \pm 0.20$ \\
\multirow{2}{*}{ Smoothing (deg) } & static & $0.23 \pm 0.02$ & $0.26 \pm 0.01$ & $0.21 \pm 0.00$ \\
& dynamic & $0.54 \pm 0.28$ & $0.36 \pm 0.25$ & $0.31 \pm 0.17$ \\
\hline
\end{tabular}

\footnotetext{
${ }^{*} r_{12}$ represents the distance between markers, refer to Fig 5. $r_{12}$ is obtained from OTS under static condition. $r_{12}$ and $r_{13}$ are approximately equal, $\left|r_{12}-r_{13}\right| \leq 2 \mathrm{~mm}$.
}

\section{B. Experiment 2-Relative Orientation Estimation Between Hand and Fingers}

The results were divided into three cases: Case I-No lineof sight occlusion; Case II-With an occlusion of markers; Case III-With a wrong identification of markers by the OTS system. The angle $\beta$ shown in Fig. 10-13 was determined by the relative orientation between hand and fingers $\boldsymbol{q}_{k}$, expressed in quaternions

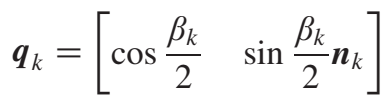

where $\beta_{k}$ is the rotation angle, $\boldsymbol{n}_{k}$ is the direction of the rotation axis.

1) Case I-No Line-of Sight Occlusion: During one trial of flexion and extension movements, markers were observable and identified correctly. The results are shown in Fig. 10. 


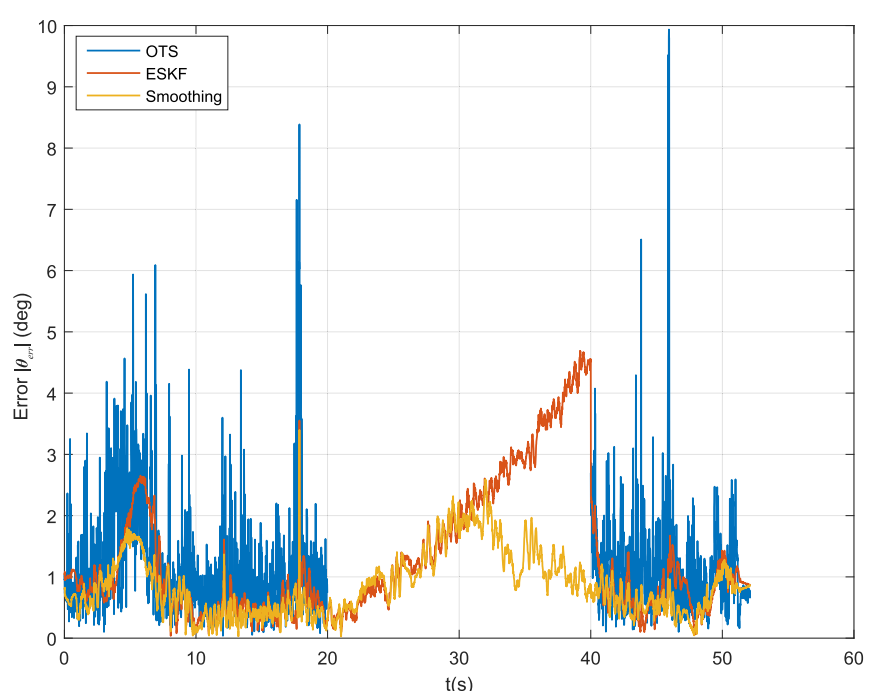

Fig. 9. Orientation estimation error with OTS separately and with EKSF fusion of OTS and gyroscope measurements, with and without additional smoothing (indicated by EKS and smoothing). The orientation error is defined in (6) and (7). During the measurement, pitch, roll, and yaw movements were performed.
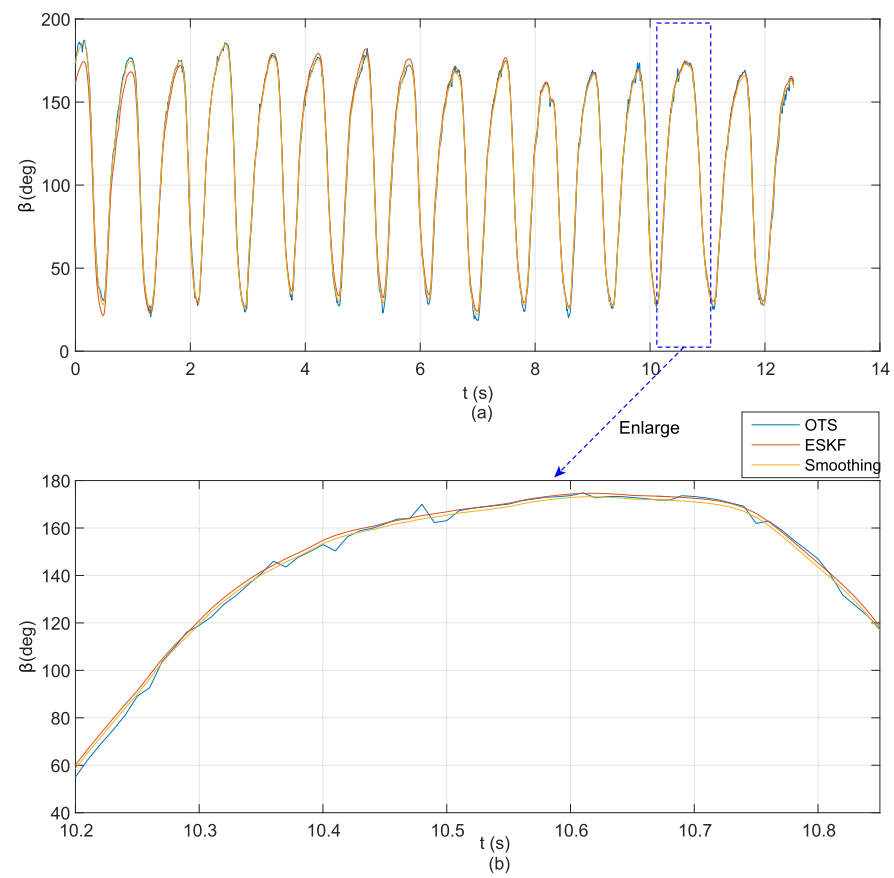

Fig. 10. Estimation of rotation angle $\beta$ during flexion and extension movements of the index finger. $\beta$ is determined by (54). During the movement, all the markers were observable and identified correctly. (a) Results during the whole movement. (b) Enlarged part of (a) between 10.2 and $10.9 \mathrm{~s}$.

Fig. 10(a) represents the flexion/extension angle $\beta$, estimated by fusing OTS and gyro information using an ESKF, and applying subsequent smoothing. The estimation based on ESKF and smoothing are smoother than the estimation based on only OTS, as shown in Fig. 10(b). Fig. 10(b) is an enlarged part of (a) between 10.2 and $10.9 \mathrm{~s}$.

During one trial of flexion and extension of the index finger, markers were not well observed, resulting in the line of sight occlusions and marker identification problems. The results are shown in Fig. 11.

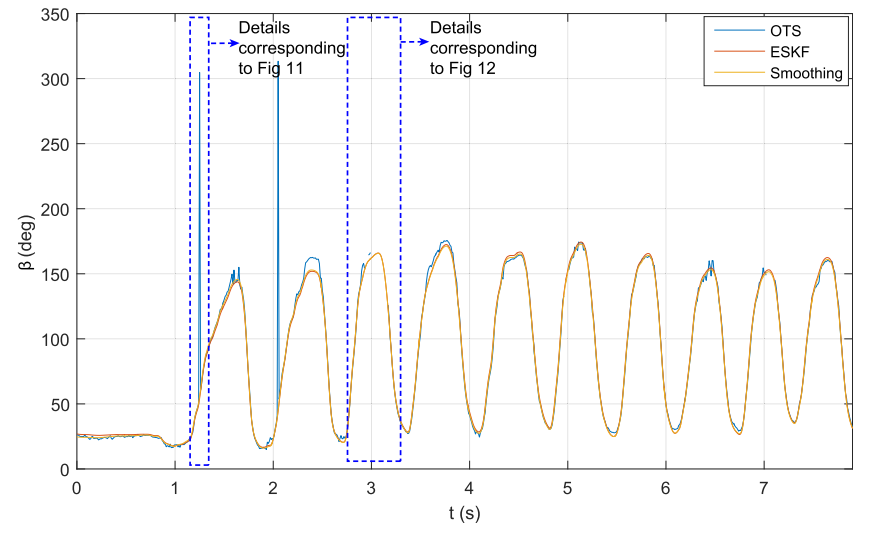

Fig. 11. Estimation of rotation angle $\beta$ during flexion and extension movements. In this trial, line of sight occlusion occurred around $3 \mathrm{~s}$ and a marker identification problem between 1.1 and $1.5 \mathrm{~s}$.

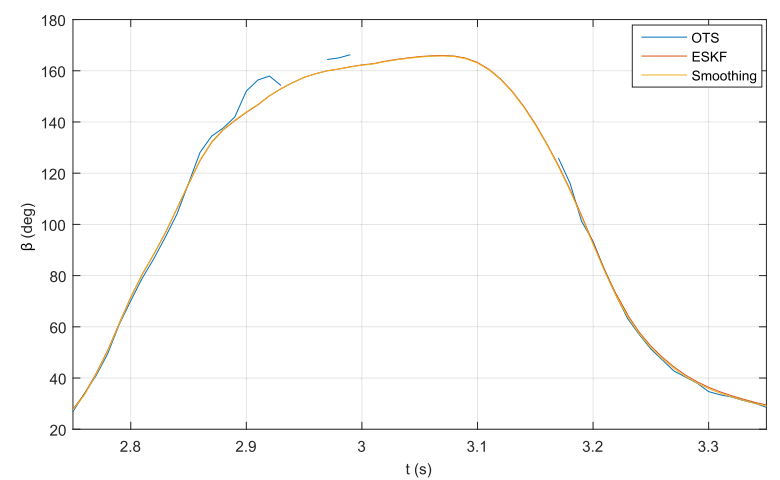

Fig. 12. Estimation of rotation angle $\beta$ during the line of sight occlusion (indicated by gaps in the blue line). This is an enlarged part of Fig. 11 during $2.7-3.4 \mathrm{~s}$.

2) Case II-With an Occlusion of Markers: Fig. 12 is an enlarged part of Fig. 11 during 2.7-3.4 s. Orientation estimation based on ESKF and smoothing appeared to be well able to fill the gap during the line of sight occlusion.

3) Case III-With a Wrong Identification of Markers by the OTS System: The OTS constantly identifies all markers during a measurement. A frequent error concerns an erroneous marker identification, for example after markers cross each other in one or several of the camera vies. This can result in sudden jumps of OTS-based 3-D orientation estimates. Fig. 13 is an enlarged part of Fig. 11 during 1.1-1.5 s. In this case, markers were observed but not correctly identified. With the OTS, relative orientation $\boldsymbol{q}_{k}$ was estimated as $-\boldsymbol{q}_{k}$, therefore $\beta$ was estimated as $304.7^{\circ}$ instead of $55.4^{\circ}$. In comparison, the ESKF and the smoothing method can provide a good estimate after fusing with gyroscope information.

\section{Discussion}

The accuracy of orientation estimation based on the OTS depends on the distance between markers. According to Wiles et al. [37], the orientation error is about $0.4^{\circ}$ with the Polaris system, Canada. The shape of the marker cluster was an isosceles right triangle and the length of the leg was $50 \mathrm{~mm}$. In our current study, the mean error changed from $2.8^{\circ}$ to $0.5^{\circ}$ when the distance between markers changed from 13 to $24 \mathrm{~mm}$. After fusing with gyroscope data, the mean error reduced to $1.5^{\circ}$ and $0.4^{\circ}$ with the ESKF. Compared with 


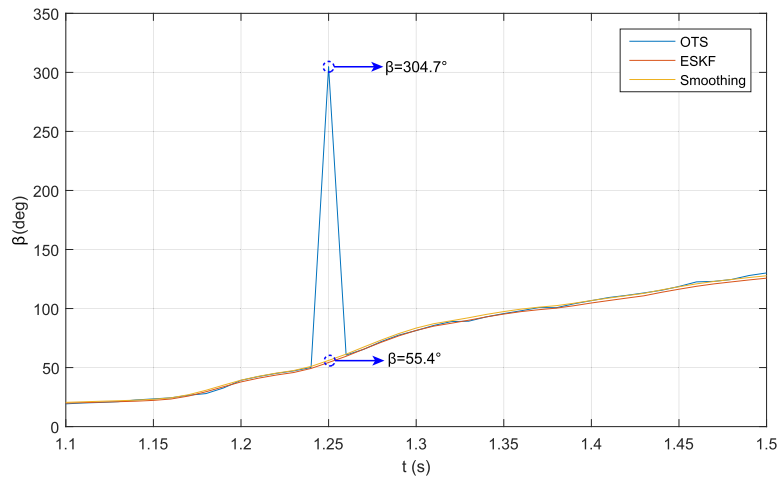

Fig. 13. Estimation of rotation angle $\beta$ in the case of a marker identification problem. This is an enlarged part of Fig. 11 at $1.25 \mathrm{~s}$.

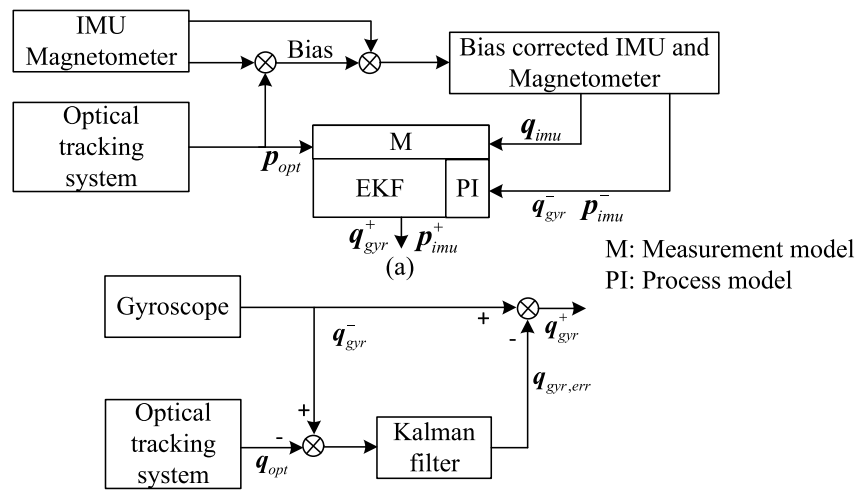

(b)

Fig. 14. Two algorithms to estimate 3-D orientation. (a) Fusion of OTS with IMU based on $\mathrm{He}$ et al. [21]. Orientation estimates are first obtained from IMU and magnetometer, and subsequently fused with OTS measurements. The initial orientation estimate is disturbed by nongravity acceleration and magnetic disturbances. (b) Our fusion method with OTS and gyroscope. It does not rely on magnetometer and accelerometer measurements.

the results of Wiles et al. [37], we achieved a comparable performance when fusing OTS and gyroscope information at half the distance between markers. This is quite useful for the applications that require high accuracy but with limited space to mount markers, such as finger segment tracking.

In previous research of He et al. [21], gyroscope, accelerometer, and magnetometer were used to improve the position and orientation accuracy of OTS. The algorithm is shown in Fig. 14(a). They used position measurements from OTS and orientation estimates from IMU and magnetometer in the measurement model to update the position and orientation estimate. However, including accelerometer and magnetometer for orientation estimation is not optimal since the inclination estimate derived from the accelerometer is disturbed by nongravity acceleration, and the heading estimate derived from magnetometer is disturbed by ferromagnetic materials and magnetic sources in the environment. By fusing with OTS position measurements, errors from the accelerometer and magnetometer can be partially reduced but cannot be eliminated. As shown in Fig. 14(b), our method does not involve an accelerometer or magnetometer. Therefore, orientation estimation errors caused by nongravity acceleration and magnetic disturbances are not present in our estimates. The estimated performances with different methods are shown in Fig. 15, the data is the same as in Fig. 8(e). During the

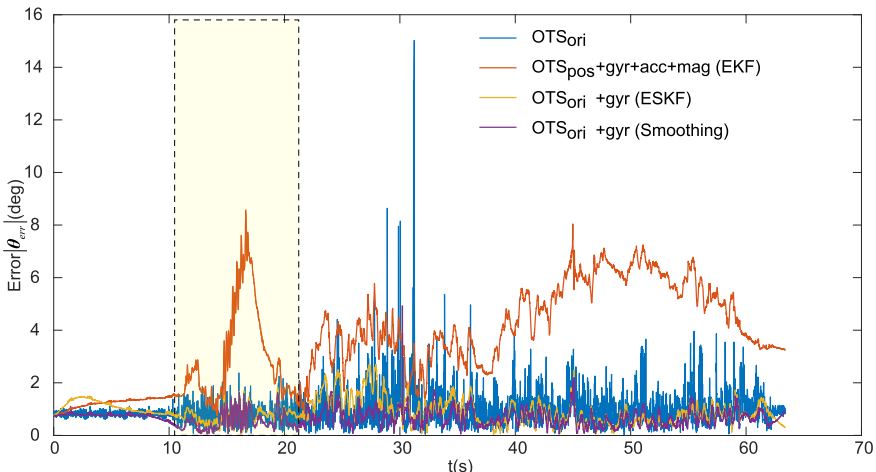

Fig. 15. Comparison of orientation estimations results with different methods. During the tests, 3-D pitch, roll, and yaw rotations were performed. The brown line represents the results with the method described by He et al. [21], which relies on the position estimation from OTS, gyroscope, accelerometer, and magnetometer. The blue line represents the result based on orientation from OTS. The yellow line represents the result based on orientation from OTS and gyroscope.

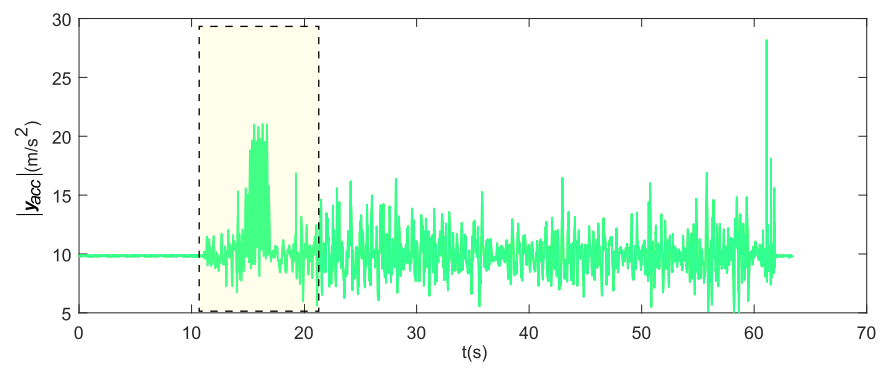

Fig. 16. Modulus of the accelerometer outputs during the movements presented in Fig. 15. During 10.1-20.2 s, there was a rapid rotation which caused a large nongravitational acceleration, the corresponding orientation estimation is shown in the shadow part of Fig. 15.

static period 0-10 s, the performances of He et al.'s method, the ESKF method, and the smoothing method are similar. During the dynamical period 10-64 s, the estimation based on He et al.'s method has a larger error than our ESKF and the smoothing method. As shown in Fig. 16, the nongravitational acceleration during 10.1-20.2 s caused a large orientation error, which can be clearly seen in Fig. 15.

The RTS smoother described in page 5 is a supplement to the proposed ESKF method, not a mandatory step. It is chosen for its efficiency and ease of implementation. Other smoothers such as Modified Bryson-Frazier smoother, minimum-variance smoother etc [38], [39] can also be applied. The limitation of RTS smoother is that it needs all the measurement data. In order to compromise between real-time use and accuracy, a smoother that only exploits the data within a window can be designed.

Our method does not solve the orientation estimation errors during a long line-of-sight occlusion. However, it can help fill short occlusion gaps in the order of 10-20 s. It should be noted that occlusion problems usually last for a few seconds. Therefore, our fusion method is a good option to improve estimation accuracy and reduce orientation estimation drift to acceptable levels during relatively short occlusion. The error can even be reduced if offline smoothing is applied (see Fig. 9). The fusion with accelerometer and magnetometer during long occlusions may improve the accuracy since they 
TABLE II

Mean Value of ERror Angle $\left|\boldsymbol{\theta}_{\text {err }}\right|$ (Deg) With Different $\kappa$ AND $\alpha$

\begin{tabular}{ccccccccc}
\hline \multirow{2}{*}{ Items } & \multicolumn{7}{c}{$\sigma_{m}(\mathrm{~mm})$} \\
\cline { 2 - 9 } & 0.02 & 0.1 & 0.18 & 0.26 & 0.34 & 0.42 & 0.5 & 0.58 \\
\hline$\alpha=15^{\circ}$ & 0.26 & 1.32 & 2.37 & 3.42 & 4.50 & 5.55 & 6.63 & 7.71 \\
$\alpha=30^{\circ}$ & 0.15 & 0.75 & 1.34 & 1.94 & 2.54 & 3.13 & 3.74 & 4.33 \\
$\alpha=45^{\circ}$ & 0.12 & 0.57 & 1.03 & 1.49 & 1.95 & 2.41 & 2.87 & 3.34 \\
$\alpha=60^{\circ}$ & 0.10 & 0.50 & 0.90 & 1.30 & 1.70 & 2.10 & 2.5 & 2.9 \\
$\alpha=75^{\circ}$ & 0.09 & 0.47 & 0.84 & 1.21 & 1.59 & 1.96 & 2.34 & 2.71 \\
$\alpha=90^{\circ}$ & 0.09 & 0.46 & 0.82 & 1.19 & 1.56 & 1.92 & 2.28 & 2.65 \\
\hline
\end{tabular}

provide disturbed orientation references in addition to only integration of angular velocity by the gyroscope.

ESKF was applied to fuse OTS and gyroscope information since it was reported to have a better performance in some areas, such as IMMU-based human movement tracking [7]. However, small drawbacks still exist. Compared with the EKF, ESKF has a more complex structure since two states need to be updated: nominal state and error state. In addition, the computational cost is higher.

\section{CONCLUSION}

An ESKF approach with an optional RTS smoother was proposed in this article to fuse orientation estimates from OTS and rate gyroscope. The objective of this approach was to improve the 3-D orientation estimation of small human body segments, like fingertips. When the proposed method was applied to a single unit of three OTS markers connected to a rate gyroscope moving in 3-D space. Two improvements were achieved. First, the accuracy of OTSbased orientation estimation was improved when markers were observable. Statically, the OTS-based orientation errors were $0.39^{\circ} \pm 0.16^{\circ}$ and $0.21^{\circ} \pm 0.03^{\circ}$ when the distances between markers were $13 \mathrm{~mm}$ and $24 \mathrm{~mm}$. These errors were reduced to $0.23^{\circ} \pm 0.02^{\circ}$ and $0.21^{\circ} \pm 0.00^{\circ}$ when applying ESKF and smoothing. under dynamic conditions, in which the orientation was continuously changed, the OTS-based orientation errors were $2.75^{\circ} \pm 1.56^{\circ}$ and $0.49^{\circ} \pm 0.34^{\circ}$ when the distances between markers were $13 \mathrm{~mm}$ and $24 \mathrm{~mm}$. This reduced to $1.50^{\circ} \pm 0.62^{\circ}$ and $0.43^{\circ} \pm 0.20^{\circ}$ with the ESKF, $0.54^{\circ} \pm 0.28^{\circ}$ and $0.31^{\circ} \pm 0.17^{\circ}$ with the additional use of the smoother. Second, ESKF and RTS smoother were able to fill gaps in OTS orientation estimates during line-of-sight occlusion. The orientation error increased to $4.8^{\circ}$ during occlusion of $20 \mathrm{~s}$ with the ESKF, which was reduced to $2.5^{\circ}$ with the RTS smoother. When the proposed method was applied to relative orientation estimation between hand and fingers, we demonstrated three advantages: first, it smoothed the orientation estimates when OTS estimation had large perturbations. Second, it filled the orientation during an occlusion period. Finally, it corrected the relative orientation estimates when the OTS wrongly identified markers.

\section{APPENDIX A}

ERroR ANGLE $\left|\boldsymbol{\theta}_{\text {err }}\right|$ (DEG) With DifFERENT $\sigma_{m}$ AND $\sigma_{m}$

For the Monte Carlo analysis corresponding to Fig. 2, the detailed value for Error angle $\left|\boldsymbol{\theta}_{\mathrm{err}}\right|$ (deg) with different $\kappa$ and $\sigma_{m}$ are specified in Table II.
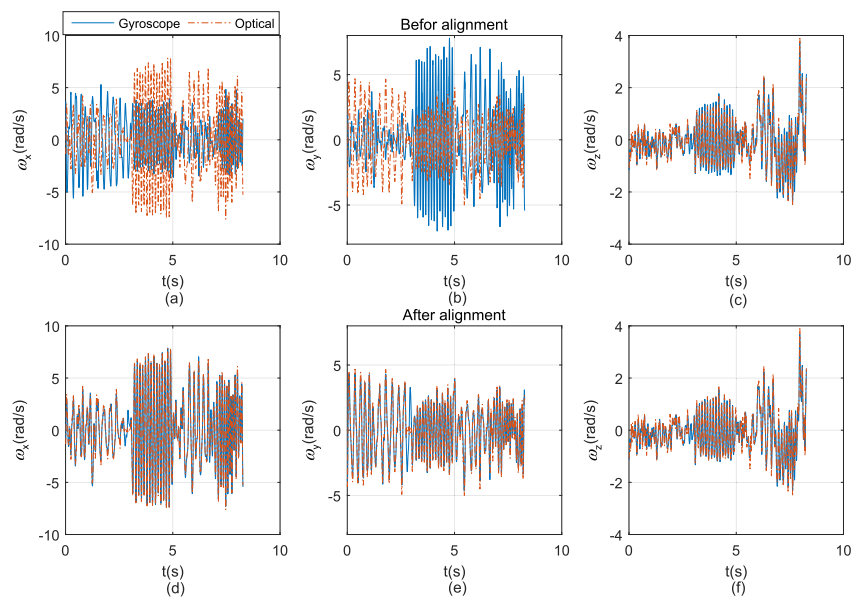

Fig. 17. Alignment results based on angular velocity. The blue lines are angular velocities measured by gyroscope, the brown lines are angular velocity estimated from OTS measurements of positions of an optical marker cluster. (a)-(c) Angular velocities before alignment. (d)-(f) Angular velocities after alignment. The angular velocities from gyroscope and optical system were both filtered with equiripple lowpass filter, the passband and stopband frequency were set as 8 and $15 \mathrm{~Hz}$ respectively.

\section{APPENDIX B}

ALIGNMENT

Fig. 17(a)-(c) shows the angular velocity from the gyroscope and OTS before alignment. (d)-(f) show the angular velocity from the gyroscope and OTS after alignment. The angular velocity difference

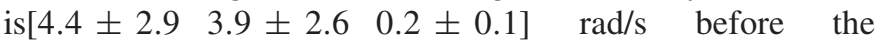
alignment. After the alignment, the difference is $\left[\begin{array}{lll}0.3 \pm 0.3 & 0.3 \pm 0.4 & 0.1 \pm 0.1\end{array}\right] \mathrm{rad} / \mathrm{s}$. The figure shows that the gyroscope and OTS estimates correspond well after the alignment.

\section{APPENDIX C PARAMETERS FOR ESKF}

For the process model, we can differentiate with respective to error-state $\boldsymbol{\theta}_{\varepsilon, k-1}, \boldsymbol{b}_{\varepsilon, k-1}$ based on (27)

$$
\begin{aligned}
& \frac{\partial\left(\boldsymbol{\theta}_{\varepsilon, k}\right)}{\partial\left(\boldsymbol{\theta}_{\varepsilon, k-1}\right)}=\left(\boldsymbol{I}_{3 \times 3}-\left\lfloor\hat{\boldsymbol{\omega}}_{k-1}^{m}\right\rfloor_{\times} d_{t}\right) \\
& \frac{\partial\left(\boldsymbol{\theta}_{\varepsilon, k}\right)}{\partial\left(\boldsymbol{b}_{\varepsilon, k-1}\right)}=-d_{t} \boldsymbol{I}_{3 \times 3} .
\end{aligned}
$$

\lfloor\rfloor$_{\times}$denotes a skew-symmetric matrix

$$
\lfloor\boldsymbol{a}\rfloor_{\times}=\left[\begin{array}{ccc}
0 & -a_{z} & a_{y} \\
a_{z} & 0 & -a_{x} \\
-a_{y} & a_{x} & 0
\end{array}\right] .
$$

Based on (36), the measurement model can be rewritten as

$$
\begin{aligned}
\overline{\boldsymbol{y}}_{k}= & \left(\hat{\boldsymbol{q}}_{g}^{s}\right)_{k} \otimes\left[\begin{array}{ll}
1 & \frac{1}{2} \boldsymbol{\theta}_{\varepsilon, k}
\end{array}\right]+\boldsymbol{n}_{\boldsymbol{y}_{k}} \\
= & \frac{1}{2}\left[\begin{array}{l}
2 \hat{q}_{a, k}-\theta_{\varepsilon_{x}, k} \hat{q}_{b, k}-\theta_{\varepsilon_{y}, k} \hat{q}_{c, k}-\theta_{\varepsilon_{z}, k} \hat{q}_{d, k} \\
2 \hat{q}_{b, k}+\theta_{\varepsilon_{x}, k} \hat{q}_{a, k}-\theta_{\varepsilon_{y}, k} \hat{q}_{d_{, k}}+\theta_{\varepsilon_{z}, k} \hat{q}_{c, k} \\
2 \hat{q}_{c, k}+\theta_{\varepsilon_{x}, k} \hat{q}_{d, k}+\theta_{\varepsilon_{y}, k} \hat{q}_{a, k}-\theta_{\varepsilon_{z}, k} \hat{q}_{b, k} \\
2 \hat{q}_{d, k}-\theta_{\varepsilon_{x}, k} \hat{q}_{c, k}+\theta_{\varepsilon_{y}, k} \hat{q}_{b, k}+\theta_{\varepsilon_{z}, k} \hat{q}_{a, k}
\end{array}\right]+\boldsymbol{n}_{\boldsymbol{y}_{k}}
\end{aligned}
$$


where $\hat{q}_{a, k}, \hat{q}_{b, k}, \hat{q}_{c, k}, \hat{d}_{d, k}, \theta_{\varepsilon_{x}, k}, \theta_{\varepsilon_{y}, k}, \theta_{\varepsilon_{z}, k}$ are components of $\left(\hat{\boldsymbol{q}}_{g}^{S}\right)_{k}$ and $\boldsymbol{\theta}_{\varepsilon, k}$.

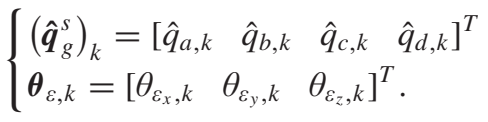

The components of Jacobian matrix $\boldsymbol{H}_{\varepsilon, k}$ in (38) are

$$
\begin{aligned}
\frac{\partial \overline{\boldsymbol{y}}_{k}}{\partial \boldsymbol{\theta}_{\varepsilon, k}}=\frac{1}{2}\left[\begin{array}{ccc}
-\hat{q}_{b, k} & -\hat{q}_{c, k} & -\hat{q}_{d, k} \\
\hat{q}_{a, k} & -\hat{q}_{d, k} & \hat{q}_{c, k} \\
\hat{q}_{d, k} & \hat{q}_{a, k} & -\hat{q}_{b, k} \\
-\hat{q}_{c, k} & \hat{q}_{b, k} & \hat{q}_{a, k}
\end{array}\right] \\
\frac{\partial \overline{\boldsymbol{y}}_{k}}{\partial \boldsymbol{\theta}_{\varepsilon, k}}=\mathbf{0}_{9 \times 3} .
\end{aligned}
$$

\section{APPENDIX D \\ DETERMINATION OF THE ORIENTATION BASED ON TWO VECTORS}

Using OTS, we obtain two vectors $\hat{\boldsymbol{r}}_{12}^{g}$ and $\hat{\boldsymbol{r}}_{13}^{g}$ from three markers. We can determine a marker frame based on these vectors and subsequently calculate the orientation of this frame with respect to the global frame in the following manner.

Step 1: Determine a normalized vector

$$
\hat{\boldsymbol{r}}_{3}^{g}=\frac{\hat{\boldsymbol{r}}_{12}^{g}}{\left|\hat{\boldsymbol{r}}_{12}^{g}\right|} .
$$

Step 2: Determine a second normalized vector based on $\hat{\boldsymbol{r}}_{3}^{g}$ and $\hat{\boldsymbol{r}}_{13}^{g}$

$$
\hat{\boldsymbol{r}}_{1}^{g}=\frac{\hat{\boldsymbol{r}}_{13}^{g} \times \hat{\boldsymbol{r}}_{3}^{g}}{\left|\hat{\boldsymbol{r}}_{13}^{g} \times \hat{\boldsymbol{r}}_{3}^{g}\right|} .
$$

Step 3: Determine a third normalized vector to formulate an orthogonal coordinate frame

$$
\hat{\boldsymbol{r}}_{2}^{g}=\frac{\hat{\boldsymbol{r}}_{3}^{g} \times \hat{\boldsymbol{r}}_{1}^{g}}{\left|\hat{\boldsymbol{r}}_{3}^{g} \times \hat{\boldsymbol{r}}_{1}^{g}\right|} .
$$

The orientation from marker to global frame can be expressed in a rotation matrix based on the above three steps

$$
\begin{aligned}
\boldsymbol{C}_{m}^{g} & =\left[\begin{array}{lll}
\hat{\boldsymbol{r}}_{1}^{g} & \hat{\boldsymbol{r}}_{2}^{g} & \hat{\boldsymbol{r}}_{3}^{g}
\end{array}\right] \\
& =\left[\begin{array}{lll}
\hat{\boldsymbol{r}}_{13}^{g} \times \hat{\boldsymbol{r}}_{3}^{g} & \hat{\boldsymbol{r}}_{3}^{g} \times \frac{\hat{\boldsymbol{r}}_{13}^{g} \times \hat{\boldsymbol{r}}_{3}^{g}}{\left|\hat{\boldsymbol{r}}_{13}^{g} \times \hat{\boldsymbol{r}}_{3}^{g}\right|} \\
\frac{\hat{\boldsymbol{r}}_{13}^{g} \times \hat{\boldsymbol{r}}_{3}^{g} \mid}{\mid \hat{\boldsymbol{r}}_{3}^{g} \times \frac{\hat{\boldsymbol{r}}_{13}^{g} \times \hat{\boldsymbol{r}}_{3}^{g} \mid}{\left|\hat{\boldsymbol{r}}_{13}^{g} \times \hat{\boldsymbol{r}}_{3}^{g}\right|}}
\end{array}\right] .
\end{aligned}
$$

The rotation matrix $\boldsymbol{C}_{m}^{g}$ can be easily transferred into quaternion $\boldsymbol{q}_{m}^{g}$ as shown in (4).

\section{ACKNOWLEDGMENT}

The authors would like to thank the Roessingh Research and Development (Enschede, Netherlands) for sharing the gait laboratory and the Lab manager, L. Schaake, for assistance using the optical system and processing of data. They would like to thank A. Droog and G. J. W. Wolterink from the Biomedical Signals and Systems research group of the University of Twente, for providing the inertial sensor setup and the 3-D printed housing for inertial sensors.

\section{REFERENCES}

[1] H. Höppner, M. Große-Dunker, G. Stillfried, J. Bayer, and P. van der Smagt, "Key insights into hand biomechanics: Human grip stiffness can be decoupled from force by cocontraction and predicted from electromyography," Frontiers Neurorobotics, vol. 11, p. 17, May 2017.

[2] D. Cervone, A. D'Amour, L. Bornn, and K. Goldsberry, "Pointwise: Predicting points and valuing decisions in real time with nba optical tracking data," in Proc. 8th MIT Sloan Sports Anal. Conf., vol. 28, Boston, MA, USA, 2014, p. 3.

[3] B. Carse, B. Meadows, R. Bowers, and P. Rowe, "Affordable clinical gait analysis: An assessment of the marker tracking accuracy of a new low-cost optical 3D motion analysis system," Physiotherapy, vol. 99, no. 4 , pp. 347-351, Dec. 2013.

[4] A. Sorriento et al., "Optical and electromagnetic tracking systems for biomedical applications: A critical review on potentialities and limitations," IEEE Rev. Biomed. Eng., vol. 13, pp. 212-232, 2020.

[5] G. Xiao et al., "Electromagnetic tracking in image-guided laparoscopic surgery: Comparison with optical tracking and feasibility study of a combined laparoscope and laparoscopic ultrasound system," Med. Phys., vol. 45, no. 11, pp. 5094-5104, Nov. 2018.

[6] M. Ota et al., "Verification of reliability and validity of motion analysis systems during bilateral squat using human pose tracking algorithm," Gait Posture, vol. 80, pp. 62-67, Jul. 2020.

[7] D. Roetenberg, C. T. M. Baten, and P. H. Veltink, "Estimating body segment orientation by applying inertial and magnetic sensing near ferromagnetic materials," IEEE Trans. Neural Syst. Rehabil. Eng., vol. 15, no. 3, pp. 469-471, Sep. 2007.

[8] J. K. Lee, E. J. Park, and S. N. Robinovitch, "Estimation of attitude and external acceleration using inertial sensor measurement during various dynamic conditions," IEEE Trans. Instrum. Meas., vol. 61, no. 8, pp. 2262-2273, Aug. 2012.

[9] Q. Yuan, E. Asadi, Q. Lu, G. Yang, and I.-M. Chen, "Uncertainty-based IMU orientation tracking algorithm for dynamic motions," IEEE/ASME Trans. Mechatronics, vol. 24, no. 2, pp. 872-882, Apr. 2019.

[10] S. O. H. Madgwick, A. J. L. Harrison, and R. Vaidyanathan, "Estimation of IMU and MARG orientation using a gradient descent algorithm," in Proc. IEEE Int. Conf. Rehabil. Robot., Jun. 2011, pp. 1-7.

[11] H. G. Kortier, V. I. Sluiter, D. Roetenberg, and P. H. Veltink, "Assessment of hand kinematics using inertial and magnetic sensors," J. NeuroEngineering Rehabil., vol. 11, no. 1, pp. 70-84, 2014.

[12] D. Roetenberg, H. J. Luinge, C. T. Baten, and P. H. Veltink, "Compensation of magnetic disturbances improves inertial and magnetic sensing of human body segment orientation," IEEE Trans. Neural Syst. Rehabil. Eng., vol. 13, no. 3, pp. 395-405, Sep. 2005.

[13] N. Yadav and C. Bleakley, "Accurate orientation estimation using AHRS under conditions of magnetic distortion," Sensors, vol. 14, no. 11, pp. 20008-20024, Oct. 2014.

[14] T. Seel, T. Schauer, and J. Raisch, "Joint axis and position estimation from inertial measurement data by exploiting kinematic constraints," in Proc. IEEE Int. Conf. Control Appl., Piscataway, NJ, USA, Oct. 2012, pp. 45-49.

[15] S. Zihajehzadeh and E. J. Park, "A novel biomechanical model-aided IMU/UWB fusion for magnetometer-free lower body motion capture," IEEE Trans. Syst., Man, Cybern. Syst., vol. 47, no. 6, pp. 927-938, Jun. 2017.

[16] T. Zimmermann, B. Taetz, and G. Bleser, "IMU-to-segment assignment and orientation alignment for the lower body using deep learning," Sensors, vol. 18, no. 1, p. 302, Jan. 2018.

[17] A. Atrsaei, H. Salarieh, and A. Alasty, "Human arm motion tracking by orientation-based fusion of inertial sensors and Kinect using unscented Kalman filter," J. Biomechanical Eng., vol. 138, no. 9, pp. 1-13, Sep. 2016

[18] Z. Zhang, "Microsoft Kinect sensor and its effect," IEEE MultimediaMag., vol. 19, no. 2, pp. 4-10, Feb. 2012.

[19] E. E. Stone and M. Skubic, "Fall detection in homes of older adults using the microsoft Kinect," IEEE J. Biomed. Health Informat., vol. 19, no. 1, pp. 290-301, Jan. 2015.

[20] R. A. Clark et al., "Validity of the microsoft Kinect for assessment of postural control," Gait Posture, vol. 36, no. 3, pp. 372-377, Jul. 2012.

[21] C. He, P. Kazanzides, H. Sen, S. Kim, and Y. Liu, "An inertial and optical sensor fusion approach for six degree-offreedom pose estimation," Sensors, vol. 15, no. 7, pp. 16448-16465, Jul. 2015. 
[22] J. Connolly, J. Condell, B. O'Flynn, J. Torres Sanchez, and P. Gardiner, "IMU sensor-based electronic goniometric glove for clinical finger movement analysis," IEEE Sensors J., vol. 18, no. 3, pp. 1273-1281, 2017.

[23] A. Rashid and O. Hasan, "Wearable technologies for hand joints monitoring for rehabilitation: A survey," Microelectron. J., vol. 88, pp. 173-183, Jun. 2019

[24] F. Kral, E. J. Puschban, H. Riechelmann, and W. Freysinger, "Comparison of optical and electromagnetic tracking for navigated lateral skull base surgery," Int. J. Med. Robot. Comput. Assist. Surg., vol. 9, no. 2, pp. 247-252, Jun. 2013

[25] D. Roetenberg, P. J. Slycke, and P. H. Veltink, "Ambulatory position and orientation tracking fusing magnetic and inertial sensing," IEEE Trans. Biomed. Eng., vol. 54, no. 5, pp. 883-890, May 2007.

[26] B. Wagstaff, V. Peretroukhin, and J. Kelly, "Robust data-driven zerovelocity detection for foot-mounted inertial navigation," IEEE Sensors J., vol. 20 , no. 2, pp. 957-967, Jan. 2020.

[27] M. B. Del Rosario, H. Khamis, P. Ngo, N. H. Lovell, and S. J. Redmond, "Computationally efficient adaptive error-state Kalman filter for attitude estimation," IEEE Sensors J., vol. 18, no. 22, pp. 9332-9342, Nov. 2018.

[28] J. Solà, "Quaternion kinematics for the error-state Kalman filter," 2017, arXiv:1711.02508. [Online]. Available: http://arxiv.org/abs/1711.02508

[29] H. M. Schepers and P. H. Veltink, "Stochastic magnetic measurement model for relative position and orientation estimation," Meas. Sci. Technol., vol. 21, no. 6, pp. 1-10, 2010.

[30] D. Weenk, D. Roetenberg, B.-J.-J. F. van Beijnum, H. J. Hermens, and P. H. Veltink, "Ambulatory estimation of relative foot positions by fusing ultrasound and inertial sensor data," IEEE Trans. Neural Syst. Rehabil. Eng., vol. 23, no. 5, pp. 817-826, Sep. 2015.

[31] M. Hao, K. Chen, and C. Fu, "Smoother-based 3-D foot trajectory estimation using inertial sensors," IEEE Trans. Biomed. Eng., vol. 66 , no. 12 , pp. 3534-3542, Dec. 2019.

[32] G. Gao, M. Kyrarini, M. Razavi, X. Wang, and A. Graser, "Comparison of dynamic vision sensor-based and IMU-based systems for ankle joint angle gait analysis," in Proc. 2nd Int. Conf. Frontiers Signal Process. (ICFSP), Oct. 2016, pp. 93-98.

[33] W.-W. Wang and L.-C. Fu, "Mirror therapy with an exoskeleton upperlimb robot based on IMU measurement system," in Proc. IEEE Int. Symp. Med. Meas. Appl., May 2011, pp. 370-375.

[34] S. SÄrkkä, "Unscented Rauch-Tung-Striebel smoother," IEEE Trans. Autom. Control, vol. 53, no. 3, pp. 845-849, Apr. 2008

[35] S. Sarkka, V. V. Viikari, M. Huusko, and K. Jaakkola, "Phase-based UHF RFID tracking with nonlinear Kalman filtering and smoothing," IEEE Sensors J., vol. 12, no. 5, pp. 904-910, May 2012.

[36] W.-L. Chan and F.-B. Hsiao, "Implementation of the Rauch-TungStriebel smoother for sensor compatibility correction of a fixedwing unmanned air vehicle," Sensors, vol. 11, no. 4, pp. 3738-3764, Mar. 2011.

[37] A. D. Wiles, D. G. Thompson, and D. D. Frantz, "Accuracy assessment and interpretation for optical tracking systems," in Medical Imaging 2004: Visualization, Image-Guided Procedures, and Display, vol. 5367. Bellingham, WA, USA: SPIE, 2004, pp. 421-432.

[38] B. K. Kwon, S. Han, O. K. Kwon, and W. H. Kwon, "Minimum variance FIR smoothers for discrete-time state space models," IEEE Signal Process. Lett., vol. 14, no. 8, pp. 557-560, Aug. 2007.

[39] R. G. Gibbs, "Square root modified Bryson-Frazier smoother," IEEE Trans. Autom. Control, vol. 56, no. 2, pp. 452-456, Feb. 2011.

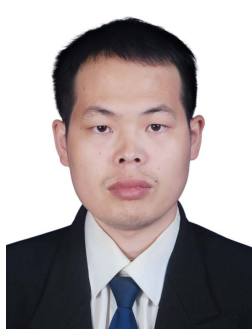

Zhicheng Yang received the B.Sc. degree in automation engineering from Zhengzhou University, in 2013. He is currently pursuing the Ph.D. degree with Northwestern Polytechnical University, Xi'an, China.

Since 2018, he has been with the University of Twente, Enschede, The Netherlands, as a joint Ph.D. degree. His main research interests include motion tracking with inertial sensors, inertial and magnetometer calibration, and localization of ferromagnetic object.

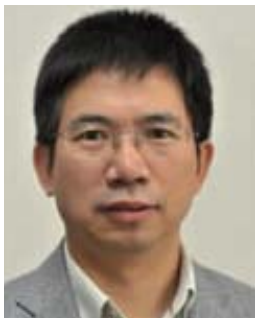

Shenggang Yan received the B.Sc. degree from Central South University in 1988 and the M.Sc. and Ph.D. degrees from Northwestern Polytechnical University, Xi'an, China, 1991 and 2008, respectively.

$\mathrm{He}$ is currently a Professor with Northwestern Polytechnical University. His main research interests include modern signal processing and its application, multisensory array, and high-speed processing.

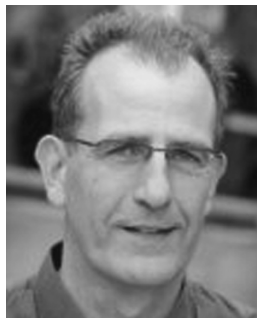

Bert-Jan F. van Beijnum (Member, IEEE) received the M.Sc. and Ph.D. degrees in electrical engineering from the University of Twente, Enschede, The Netherlands.

$\mathrm{He}$ is currently an Associate Professor with the Remote Monitoring and Treatment Section, Bio Signals and Systems Research Group, University of Twente, Enschede. His research focus is on health systems for chronic diseases and well-being, and his research interests include telemedicine, mobile virtual communities for health, smart health systems, smart technologies for decision support, feedback, and coaching.

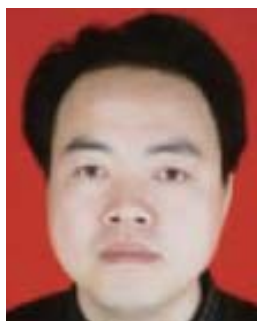

Bin $\mathbf{L i}$ received the B.Sc. and M.Sc. degrees from Northwestern Polytechnical University, Xi'an, China, 1985 and 1988, respectively.

$\mathrm{He}$ is currently a Professor with Northwestern Polytechnical University. His main research interest includes magnetic target detection, sensor technology, and marine electromagnetic field communication.

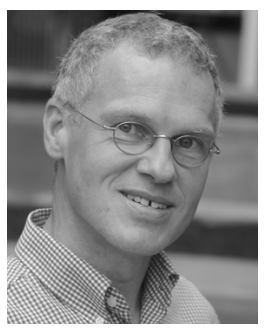

Peter H. Veltink (Senior Member, IEEE) is currently a Professor of technology for the restoration of human function with the University of Twente, Enschede, The Netherlands. He is the (co)author of over 125 peer reviewed journal articles. He was the Scientific Coordinator of three EU research training networks. He had also coordinates the EU project Interaction. His research interests are in biomechatronics and neural engineering. His research includes ambulatory sensing of human movements, artificial human motor control, and neurostimulation. Prof. Veltink has been an Associate Editor for the IEEE TRANSACTIONS of NeURAL Systems AND REHABILITATION ENGINEERING until 2014. 